\title{
Robust a posteriori stress analysis for quadrature collocation approximations of nonlocal models via nonlocal gradients
}

\author{
Qiang Du ${ }^{\mathrm{a}}$, Yunzhe $\mathrm{Tao}^{\mathrm{a}}$, Xiaochuan Tian ${ }^{\mathrm{a}}$, Jiang Yang ${ }^{\mathrm{a}}$ \\ ${ }^{a}$ Department of Applied Physics and Applied Mathematics, Columbia University, New York, \\ NY 10027
}

\begin{abstract}
As alternatives to partial differential equations (PDEs), nonlocal continuum models given in integral forms avoid the explicit use of conventional spatial derivatives and allow solutions to exhibit desired singular behavior. It is of practical interest to develop robust numerical schemes not only for the numerical solution of nonlocal models but also for the evaluation of suitably defined derivatives of solutions. The latter motivates the development of a nonlocal analog of gradient recovery for numerical solution of PDEs. For structure mechanical models, this leads to a posteriori nonlocal stress analysis. We illustrate that when smooth solutions are found in nonlocal models, one may compute local gradients of nonlocal solutions using conventional techniques like that for PDEs. More generically however, we present a framework for stress analysis of nonlocal solutions based on nonlocal gradient operators and their asymptotically compatible discretization. We demonstrate that the nonlocal gradient recovery is consistent in the local limit and is more advantageous than using local gradients of nonlocal solutions. Superconvergence properties of some special nonlocal gradient operators are identified for nonlocal continuum models. Moreover, methods are presented to preserve such features in the numerical discretization. Both computational observations and theoretical insights are provided to substantiate our findings.
\end{abstract}

Keywords: Nonlocal gradient, nonlocal stress, nonlocal models, peridynamics, asymptotic compatibility, quadrature collocation approximations, order of convergence, superconvergence

\footnotetext{
This work is supported in part by the U.S. NSF grant DMS-1318586, and AFOSR MURI center for material failure prediction through peridynamics.

Email addresses: qd2125@columbia.edu (Qiang Du), y.tao@columbia.edu (Yunzhe Tao), xt2156@columbia.edu (Xiaochuan Tian), jy2759@columbia.edu (Jiang Yang)
} 


\section{Introduction}

This work is concerned with computational studies of nonlocal models that use integral formulations to represent nonlocal spatial interactions. By avoiding the explicit use of spatial derivatives, a nonlocal model can potentially allow more singular solutions than its classical differential equation counterpart. This can become a great advantage in the modeling of complex systems such as those involving material interfaces and defects. Indeed, there have been many recent works in which nonlocal models are used to study problems that arise in physics, biology, materials and social sciences [5, 7, 3, 40, 9, 18, 23]. A particular motivation for our work is the increasing interest in the nonlocal peridynamics (PD) continuum theory introduced first by Silling [34]. PD has been applied to study cracks and materials failure as well as other mechanical properties and physical processes [4, 29, 36, 37]. Along with successful applications, rigorous mathematics of PD like nonlocal models has also been developed $[2,17]$. In $[11,12]$, an attempt is made to develop a systematic mathematical framework for nonlocal problems, in parallel to that for local classical partial differential equations (PDEs). As exact solutions of nonlocal models are not feasible in practice, effective numerical methods can play an important role in the study of these models $[8,10,19,24,32,35,46]$.

A distinct feature shared among nonlocal models such as peridynamics is that the range of nonlocal interactions is bounded by a positive parameter, the so called horizon parameter $\delta$ [34]. For suitably defined nonlocal interaction kernels, as $\delta \rightarrow 0$, interactions can become localized so that the zero-horizon limit of the nonlocal operators, when it exists, becomes a local differential operator. That is, a nonlocal model gets reduced to a conventional differential equation model in the local limit. The local limit may be derived formally for smooth solutions. It can also be rigorously established for nonlocal solutions having only minimal regularity, see for instance the convergence analysis of linear state-based PD models to the Navier equation of elasticity [26]. A discretization scheme that preserves this limiting behavior is called an asymptotically compatible scheme, a notion developed in $[42,43]$. In other words, given a simulation code designed for nonlocal models based on an asymptotically compatible scheme, by taking a sufficiently small horizon parameters in the code, one may recover, from the computed nonlocal solutions, their local limit solution if the local model and its solution are physically valid and mathematically well-defined. This is a useful property when performing bench-marking studies using simulation codes for nonlocal models, as the consistency with known local solutions is often the first step of code validation and verification.

Another aspect of nonlocal formulations of mechanics such as peridynamics is that the equations are posed in terms of displacement or displacement field. In applications like structure analysis, the primary interest might be in the deformation gradient or, through appropriate constitutive relation, the stress, rather than the deformation itself. For traditional PDEs models, various numerical techniques have been developed in order to achieve accurate evalu- 
ations of solution derivatives, such as local gradient recovery (mostly done by a posteriori error estimation [45]) and mixed methods (solving for deformation gradient or stress variables directly $[25,31]$ ). Nonlocal peridynamics is seen as a derivative-free formulation which makes it adapt to the nucleation and propagation of singularities and jumps in deformation fields. Until present, there has not been any mixed method formulation for nonlocal peridynamics using local spatial gradients or conventional stresses. Although the PD stress tensor has been introduced [34], gradient recovery techniques for nonlocal models remain largely unexplored in the literature. In this work, we make some preliminary investigations on the latter. Our study leads to the development of accurate and robust numerical schemes not only for nonlocal solutions but also their suitably defined or properly interpreted nonlocal spatial derivatives.

The central theme of this paper is to promote the application of asymptotically compatible (AC) schemes in both the numerical solution of nonlocal models and the a posteriori stress analysis. The application is seen in two phases: finding the solution first, followed by the post-processing of solution. First in the solving phase, we recall that, according to $[42,43]$ where AC schemes are first proposed and studied, an AC scheme assures accurate and robust determination of nonlocal solutions that, for sufficiently small horizon parameters, are compatible with solutions of the limiting local PDE models, whenever the latter are physically valid and mathematically well-posed. Once the nonlocal solutions are found, we are in the post-processing phase that includes, for much of this work, the task of stress analysis or gradient recovery. While for smooth nonlocal solutions one may use conventional gradient recovery techniques like those developed for PDEs, such a practice may not be effective for more generic situations where nonlocal solutions of practical interest may not be regular. Indeed, nonlocal models are often designed to capture singular or discontinuous solutions such as cracks and other materials defects modeled by PD models. Hence, our main message is that nonlocal stress analysis based on nonlocal gradient operators and their asymptotically compatible discretization is much more effective than the conventional local gradient recoveries. Such nonlocal gradient recovery and nonlocal stress analysis are new contributions to the subject of nonlocal modeling and simulations and will likely impact practical applications on nonlocal models. To illustrate the generality, we consider the quadrature collocation approximations based on a strong form of the nonlocal models, and discuss different forms of nonlocal gradients. The quadrature collocation approximation is chosen due to the popularity of method based on strong form such as the original meshfree method developed in [34]. As this is the first study in this direction, in order to better illustrate the key ideas, we focus on one-dimensional examples and present a comparison of the local gradients with a couple of different forms of nonlocal gradients. In particular, we explore some interesting superconvergence properties associated with the special onedimensional case considered in this work. More in-depth studies and analysis in higher dimensions and applications to nonlinear models are to be carried out in separate works. 
The remaining of the paper is organized as follows. We provide a brief discussion in section 2 on a model problem defined on a one dimensional bar and its asymptotically compatible quadrature collocation approximations. Nonlocal gradient operators are reviewed with some particular forms identified for this work. Their discrete forms and associated properties are also presented. Then, we report several carefully designed numerical experiments to illustrate the effective use of nonlocal stress analysis in section 3 . We also provide additional theoretical justifications to our findings in section 4. Finally, in section 5, we make some conclusions on the general use of nonlocal gradients in nonlocal modeling.

\section{Nonlocal model and quadrature collocation approximation}

In this section, we discuss how nonlocal gradient operators can offer a more robust tool for nonlocal stress analysis, in comparison with the use of conventional local gradients and local differential operators. Our discussion here is focused on quadrature/collocation approximations of nonlocal models, though much of the analysis works for other approximation methods as well.

\subsection{Nonlocal model}

To illustrate the key concepts, we present the study for a linear nonlocal model defined on a finite bar $\Omega$ in $\mathbb{R}$. To further avoid unnecessary complications due to nonlocal boundary conditions (that is, nonlocal volume constraints, see $[11,12]$ for more detailed discussion), periodic condition is imposed with the periodic cell given by $\Omega=(0,1)$. The simple setting allows us to compare with results of their well-defined limiting local models. It is expected that our approach works also for systems of equations in multi-dimensional spaces.

Let $u=u(x)$ denote a scalar deformation field. We consider a nonlocal operator $\mathcal{L}_{\delta}$ defined by

$$
\mathcal{L}_{\delta} u(x)=2 \int_{-\delta}^{\delta} \rho_{\delta}(s)(u(x+s)-u(x)) d s,
$$

where $\delta>0$ denotes the nonlocal horizon parameter and $\rho_{\delta}(s)$ is a nonlocal interaction kernel to be specified later. The nonlocal balance law is prescribed by

$$
-\mathcal{L}_{\delta} u=f \quad \text { on } \Omega,
$$

for a given external force $f$ satisfying the compatibility condition

$$
\int_{\Omega} f(x) d x=0 .
$$


For illustration, we consider suitably scaled kernels so that the local limit of nonlocal operators $\mathcal{L}_{\delta}$ as $\delta$ goes to zero is exactly the second order derivative $\frac{d^{2}}{d x^{2}}$ which is denoted by $\mathcal{L}_{0}$. A popular choice is given by

$$
\rho_{\delta}(s)=\frac{1}{\delta^{3}} \rho\left(\frac{|s|}{\delta}\right), \quad \forall s \in[-\delta, \delta], \quad \rho_{\delta}(s)=0, \quad \forall|s|>\delta .
$$

where the symmetric kernel $\rho=\rho(\xi)$ is a nonnegative nonincreasing function with a compact support in $[0,1]$ and a normalized second moment, i.e.,

$$
\left\{\begin{array}{c}
\rho(\xi)=0, \quad \forall \xi>1, \quad \rho^{\prime}(\xi) \leq 0, \quad \forall \xi \in(0,1), \\
\rho(\xi)=\rho(-\xi), \forall \xi \in \mathbb{R}, \quad \int_{\mathbb{R}} \rho(\xi) \xi^{2} d \xi=1 .
\end{array}\right.
$$

An additional constraint is needed to make the solution unique. We take

$$
\int_{\Omega} u(x) d x=0
$$

for simplicity. The limiting local model of (2.2) is then given by

$$
-\mathcal{L}_{0} u=f \quad \text { in } \Omega=(0,1),
$$

for $\mathcal{L}_{0}=\frac{d^{2}}{d x^{2}}$, again with the periodic condition and uniqueness condition imposed in (2.5).

\subsection{Nonlocal gradient operator}

The operator $\mathcal{L}_{\delta}$ can be seen as the nonlocal analog of the second order derivative $\mathcal{L}_{0}$. It is natural to introduce a nonlocal analog of the first order derivative, or the nonlocal gradient operator in multidimensions. Detailed studies of these operators are the subject of recently developed nonlocal vector calculus and we refer to [11] for formal derivations and [27] for more extended functional analysis. Nonlocal analog of integration by parts formula has also been rigorously derived [16, 27].

Here, to recall the necessary definitions, we limit ourselves to the nonlocal gradient operators defined below:

$$
\mathbb{G}_{\delta} u(x)=\int_{-\delta}^{\delta} s \beta_{\delta}(s)(u(x+s)-u(x)) d s,
$$

where $\beta_{\delta}=\beta_{\delta}(s)$ is a nonnegative kernel with a bounded second moment. In particular, it may be taken as a rescaled form $\beta_{\delta}(s)=\delta^{-3} \beta(s / \delta)$, the same as (2.3), with $\beta$ sharing the same properties given in (2.4). Another way of looking 
at $\mathbb{G}_{\delta}$ is to rewrite $(2.7)$ as

$$
\begin{aligned}
\mathbb{G}_{\delta} u(x) & =\int_{-\delta}^{\delta} s^{2} \beta_{\delta}(s) \frac{u(x+s)-u(x)}{s} d s \\
& =\int_{-\delta}^{\delta} s^{2} \beta_{\delta}(s) \frac{u(x)-u(x-s)}{s} d s \\
& =\int_{-\delta}^{\delta} s^{2} \beta_{\delta}(s) \frac{u(x+s)-u(x-s)}{2 s} d s .
\end{aligned}
$$

With the assumptions on $\beta_{\delta}(s)$, we may view $s^{2} \beta_{\delta}(s)$ as a density function, so that $\mathbb{G}_{\delta}$ is effectively a continuum weighted average of some discrete first order difference operators up to the scale $\delta$. If $\delta \rightarrow 0, s^{2} \beta_{\delta}(s)$ gets localized and behaves like a Dirac-delta measure at the origin, thus, we indeed have $\frac{d}{d x}$ as the local limit of $\mathbb{G}_{\delta}$.

The above definition is a scalar version of a specialized case of nonlocal gradient operators defined in [11] and [27]. There are also the one sided versions (see $[41,16]$ for related discussions)

$$
\mathbb{G}_{\delta}^{ \pm} u(x)= \pm 2 \int_{0}^{\delta} s \beta_{\delta}(s)(u(x \pm s)-u(x)) d s,
$$

that one may also consider, in particular in the presence of geometric boundary or potential discontinuities.

We note that there have been much discussions on nonlocal peridynamic stresses and nonlocal strains in the literature, see [34, 21] and [6, 44]. For the one dimensional nonlocal operator $\mathcal{L}_{\delta}$ associated with a kernel $\rho_{\delta}$, a particular nonlocal gradient operator $\mathbb{G}_{\delta}^{p}$ is given by

$$
\mathbb{G}_{\delta}^{p} u(x)=\int_{-\delta}^{\delta} s \gamma_{\delta}(s)(u(x+s)-u(x)) d s,
$$

for any scalar field $u=u(x)$, and

$$
\gamma_{\delta}(s)=\frac{2}{s} \int_{s}^{\delta} \rho_{\delta}(z) d z, \text { for } 0<s \leq \delta, \quad \gamma_{\delta}(s)=0, \text { for } s>\delta,
$$

and $\gamma_{\delta}(s)=\gamma_{\delta}(-s)$ for $s<0$.

A direct calculation gives

$$
-\frac{d}{d x} \mathbb{G}_{\delta}^{p} u(x)=-\mathcal{L}_{\delta} u(x)
$$

where $\mathcal{L}_{\delta}$ is given by (2.1). The equation (2.10) is consistent with a derivation of the nonlocal peridynamic stress given in [21] for a deformation field in multiple space dimensions that has been seen as a consequence of the Noll's lemma, hence 
the use of superscript $p$ in the notation $\mathbb{G}_{\delta}^{p}$. Our formulation adopts a simpler and more conventional representation in the same form as (2.7). we note that for $\rho_{\delta}$ satisfying (2.3) and (2.4), the function $\gamma_{\delta}$ also shares the same properties as it can be written by

$$
\gamma_{\delta}(s)=\frac{2}{s} \int_{s}^{\delta} \frac{1}{\delta^{3}} \rho\left(\frac{z}{\delta}\right) d z=\frac{1}{\delta^{3}} \frac{2 \delta}{s} \int_{s / \delta}^{1} \rho(z) d z=\frac{1}{\delta^{3}} \gamma\left(\frac{s}{\delta}\right),
$$

where for $s \geq 1$, and $\gamma(s)=0$ for $s \in(0,1)$,

$$
\gamma(s)=\frac{2}{s} \int_{s}^{1} \rho(z) d z, \quad \gamma^{\prime}(s)=-\frac{2}{s^{2}} \int_{s}^{1} \rho(z) d z-\frac{2}{s} \rho(s) \leq 0 .
$$

It is also easy to check that

$$
\int_{\mathbb{R}} \gamma(|\xi|) \xi^{2} d \xi=2 \int_{0}^{1} s^{2} \rho(s) d s=1 .
$$

In some applications, the equation (2.2) may also represent a nonlocal diffusion equation where the nonlocal derivative or gradient, such as that given by $\nu=\mathbb{G}_{\delta}^{p} u(x)$, may be called a nonlocal flux. For more discussions on the latter, we refer to [11].

For the simple 1d linear model (2.2) with a periodic boundary condition (so that boundary effect can be ignored), and the nonlocal solution $u_{\delta}=u_{\delta}(x)$ of (2.2) and the local limit solution $u_{0}=u_{0}(x)$ of (2.6) with the same right hand side function $f=f(x)$, it has been shown [15] that the difference between $u_{\delta}$ and $u_{0}$ are in general on the order of $O\left(\delta^{2}\right)$, see similar estimates in Theorem 4 in Section 4. However, by taking the first integrals of both the nonlocal and local equations, we can derive from (2.10) the following result.

Theorem 1. For the same data $f=f(x)$, let $u_{\delta}$ be the solution of the nonlocal equation (2.2) and $u_{0}$ be the solution of the local limit (2.6). Then

$$
\mathbb{G}_{\delta}^{p} u_{\delta}(x)=\frac{d}{d x} u_{0}(x) .
$$

The above relation implies the superconvergence or exactness of the nonlocal gradient of the nonlocal solution to the local derivative of the associated local limit solution. This, of course, is a rather special consequence valid for the $1 \mathrm{~d}$ problems under consideration. More general multidimensional cases will be studied in separate works. For other choices of the kernel $\beta_{\delta},(2.11)$ may not hold, and the error $\mathbb{G}_{\delta} u_{\delta}-\frac{d}{d x} u_{0}$ might depend on $\delta$, the nonlocal kernels and the data $f$. More detailed discussions can be found in later sections.

While $\mathbb{G}_{\delta}^{p}$ has nice properties like (2.11), we note that nonlocal gradients given in (2.7) and (2.8) provide us more choices. For instance, the one-sided version may be useful near the boundary in the presence of other boundary conditions. It can also help to model situations that no longer have the forwardbackward symmetry [16]. 


\subsection{Quadrature collocation scheme}

For a positive integer $N$, we set $h=1 / N$ and $\delta=r h+\delta_{0}$ for a nonnegative integer $r<N$ and $\delta_{0} \in[0, h)$. We denote the quadrature points on $\Omega$ by $\left\{x_{i}=i h\right\}_{i=0}^{N}$ and follow the convention that $x_{j}=x_{\bmod (j, N)}$. The same periodic extension convention is applied to other relevant vector arrays such as the

discrete solutions. A standard continuous piecewise linear hat basis function is given by

$$
\phi_{i}^{1}(x)= \begin{cases}\left(x-x_{i-1}\right) /\left(x_{i}-x_{i-1}\right) & \text { for } x \in\left(x_{i-1}, x_{i}\right) \\ \left(x_{i+1}-x\right) /\left(x_{i+1}-x_{i}\right) & \text { for } x \in\left[x_{i}, x_{i+1}\right) \\ 0, \quad \text { otherwise } & \end{cases}
$$

We adopt the quadrature collocation scheme studied in [42]: for $i=1, \ldots, N$,

$$
\begin{aligned}
-\mathcal{L}_{\delta, 1}^{h} u_{i}= & -2 \sum_{m=1}^{r} \frac{u_{i-m}-2 u_{i}+u_{i+m}}{(m h)} \int_{I_{m} \cup I_{m+1}} \phi_{m}^{1}(s) s \rho_{\delta}(s) d s \\
& -\frac{2\left(u_{i-r-1}-2 u_{i}+u_{i+r+1}\right)}{(r+1) h} \int_{I_{r+1}} \phi_{r+1}^{1}(s) s \rho_{\delta}(s) d s=f_{i},
\end{aligned}
$$

where $I_{j}=((j-1) h, j h)$ for $1 \leq j \leq r$, and $I_{r+1}=(r h, \delta)$.

The scheme (2.13) is asymptotically compatible (AC) [42] in the sense that for a given finite $\delta$, as $h \rightarrow 0$, we have the convergence of the numerical solution (2.13) to the solution of the nonlocal model (2.2). At the same time, if both $\delta$ and $h$ are approaching 0 simultaneously, then we get the convergence to the solution of the local limit (2.6). AC property is important for the validation of nonlocal models when performing bench-mark tests against traditional local models, if the latter are applicable and well-defined. For further discussions on the convergence and asymptotic compatibility of the (2.13), we refer to [42] and [14].

Let us use $\mathbb{A}_{\delta}^{h}$ to denote the quadrature collocation matrix associated with horizon $\delta$ and mesh spacing $h$ and let $\mathbb{A}^{h}$ correspond to the coefficient (stiffness) matrix associated with the discretization of local operator. Similar to [42], the stiffness matrix $\mathbb{A}_{\delta}^{h}$ is a symmetric Toeplitz matrix with first row

$$
\mathbb{A}_{\delta}^{h}(1,:)=-\left(b_{0}, b_{1}, \cdots, b_{r+1}, 0, \cdots, 0, b_{r+1}, b_{r}, \cdots, b_{1}\right),
$$


where

$$
-b_{m}:=\left\{\begin{array}{lr}
\frac{4}{h} \sum_{k=1}^{r} \int_{I_{k} \cup I_{k+1}} \frac{\phi_{k}^{1}(s)}{k} s \rho_{\delta}(s) d s+\frac{4}{h} \int_{I_{r+1}} \frac{\phi_{r+1}^{1}(s)}{r+1} s \rho_{\delta}(s) d s, & m=0 \\
-\frac{2}{m h} \int_{I_{m} \cup I_{m+1}} \phi_{m}^{1}(s) s \rho_{\delta}(s) d s, & 1 \leq m \leq r \\
-\frac{2}{(r+1) h} \int_{I_{r+1}} \phi_{r+1}^{1}(s) s \rho_{\delta}(s) d s, & m=r+1 \\
0, & \text { otherwise. }
\end{array}\right.
$$

Similar to the approximation to $\mathcal{L}_{\delta}$, we may consider the following discretization of the nonlocal gradient operator: for $i=1, \ldots, N$,

$$
\begin{aligned}
\mathbb{G}_{\delta, h} u_{i}= & \sum_{m=1}^{r}\left(u_{i+m}-u_{i-m}\right) \int_{I_{m} \cup I_{m+1}} \phi_{m}^{1}(s) s \beta_{\delta}(s) d s \\
& +\left(u_{i+r+1}-u_{i-r-1}\right) \int_{I_{r+1}} \phi_{r+1}^{1}(s) s \beta_{\delta}(s) d s
\end{aligned}
$$

We may also consider the one sided versions of $\mathbb{G}_{\delta, h}$, the details are omitted. In comparison, we let $\mathbb{G}_{0, h}^{+}, \mathbb{G}_{0, h}^{-}$and $\mathbb{G}_{0, h}$ denote the local discrete derivative operators with forward, backward and central differences respectively, namely

$$
\mathbb{G}_{0, h}^{ \pm} u_{j}= \pm \frac{u_{j \pm 1}-u_{j}}{h}, \quad \text { and } \quad \mathbb{G}_{0, h} u_{j}=\frac{u_{j+1}-u_{j-1}}{2 h} .
$$

One may note that the above discretization $\mathbb{G}_{\delta, h}$ also provides an asymptotically compatible approximation to the nonlocal gradient operator and its local limit, as demonstrated through theoretical analysis presented later.

Now, given the freedom in the choices of the kernels for the nonlocal gradient, let us also consider a discrete analog of $\mathbb{G}_{\delta}^{p}$. For this, we let $c_{m}=h^{2} b_{m}$ and $S$ denote the forward shift operator, that is $S u_{j}=u_{j+1}$, then we have

$$
\begin{aligned}
\mathcal{L}_{\delta, 1}^{h} & =\frac{1}{h^{2}} \sum_{m=1}^{r+1}\left(S_{-m}-2 I+S_{m}\right) c_{m} \\
& =\frac{1}{h^{2}}(S-I)\left(I-S^{-1}\right) \sum_{m=1}^{r+1} c_{m}\left(\sum_{k=0}^{m-1} S^{k}\right)\left(\sum_{k=0}^{m-1} S^{-k}\right) \\
& =\frac{1}{h^{2}}(S-I)\left(I-S^{-1}\right) \mathbb{Y}_{\delta, h} \\
& =\frac{1}{h}(S-I) \mathbb{G}_{\delta, h}^{p-} \\
& =\frac{1}{h}\left(I-S^{-1}\right) \mathbb{G}_{\delta, h}^{p+}
\end{aligned}
$$


where the operators $\mathbb{G}_{\delta, h}^{p \pm}$ and $\mathbb{Y}_{\delta, h}$ are given respectively by

$$
\mathbb{Y}_{\delta, h}=\sum_{m=1}^{r+1} c_{m}\left(\sum_{k=0}^{m-1} S^{k}\right)\left(\sum_{k=0}^{m-1} S^{-k}\right), \quad \mathbb{G}_{\delta, h}^{p \pm}= \pm \frac{1}{h}\left(S^{ \pm 1}-I\right) \mathbb{Y}_{\delta, h} .
$$

Then by noting that $(S-I)\left(I-S^{-1}\right) / h^{2}$ is in fact the second order central difference of (2.6), we have derived the following special relation.

Theorem 2. Let $u_{\delta, h}$ and $u_{0, h}$ be the solutions of (2.13) and the central second order difference scheme of (2.6), then

$$
\mathbb{Y}_{\delta, h} u_{\delta, h}=u_{0, h}, \quad \mathbb{G}_{\delta, h}^{p \pm} u_{\delta, h}=\mathbb{G}_{0, h}^{ \pm} u_{0, h}
$$

and consequently,

$$
\mathbb{G}_{\delta, h}^{p} u_{\delta, h}=\frac{1}{2}\left(\mathbb{G}_{\delta, h}^{p-}+\mathbb{G}_{\delta, h}^{p+}\right) u_{\delta, h}=\mathbb{G}_{0, h} u_{0, h} .
$$

The above result in particular implies the superconvergence, in fact, the exactness at the discrete level, of the nonlocal discrete gradients of the nonlocal solution to the local discrete gradients (forward, backward and central differences) of the local solution. A further consequence of the exactness of the nonlocal gradient and its discrete approximation to the respective local limits is the error estimate between $\mathbb{G}_{\delta, h}^{p} u_{\delta, h}$ and $\mathbb{G}_{\delta}^{p} u_{\delta}$, which exhibits uniform convergence independent of the parameter $\delta$.

Theorem 3. Let $u_{\delta}$ be the solution of the nonlocal equation (2.2) and $u_{\delta, h}$ be the solution of quadrature approximation (2.13). Let $u_{0}$ and $u_{0, h}$ be the solution of (2.6) and the central second order difference approximation. Then,

$$
\mathbb{G}_{\delta, h}^{p} u_{\delta, h}-\mathcal{R}_{h} \mathbb{G}_{\delta}^{p} u_{\delta}=\mathbb{G}_{0, h} u_{0, h}-\mathcal{R}_{h} \frac{d u_{0}}{d x}
$$

where $\mathcal{R}_{h}$ denotes the restriction operator with respect to the quadrature points $\left\{x_{i}=i h\right\}_{i=0}^{N}$, that is, $\mathcal{R}_{h}$ takes a function defined on the interval to a vector whose entries are given by the function values at $\left\{x_{i}=i h\right\}_{i=0}^{N}$.

With the periodic boundary condition, it is easy to see that the order of the above error in $h$ is contingent upon the regularity of the exact solution of the local equation that in fact is determined by the regularity of the right hand side $f=f(x)$. For nonlocal gradient $\mathbb{G}_{\delta}$ and the one-sided versions $\mathbb{G}_{\delta}^{ \pm}$with a more generic kernel $\beta_{\delta}$, the above superconvergence may not always hold. The orders of numerical errors will be examined more closely in the experiments and analysis presented later. We note that one can carry out similar discussions if the nonlocal model is discretized using Galerkin finite element methods, details will be presented in a separate work. 


\section{Numerical experiments}

In this section, we present a set of numerical experiments to illustrate the application of nonlocal gradient recovery. For convenience, we work with a simple piecewise constant kernel

$$
\rho_{\delta}(s)=\frac{3}{2 \delta^{3}} \chi_{[-\delta, \delta]}(s),
$$

where $\chi$ is the characteristic function. We let $\beta_{\delta}=\rho_{\delta}$ in the nonlocal operator $\mathbb{G}_{\delta}$ and use the $\mathrm{AC}$ quadrature discretization discussed earlier. In comparison, we also present nonlocal gradients of the special operator $\mathbb{G}_{\delta}^{p}$. In this case,

$$
\gamma_{\delta}(s)=\frac{3(\delta-|s|)}{|s| \delta^{3}} \chi_{[-\delta, \delta]}(s),
$$

and we adopt (2.16) and (2.17) as numerical discretization. In order to get nonlocal solutions with different regularities, we choose a right hand side $f$ that is characterized by prescribed positive parameters $\sigma$ and $k$.

For the first example, we consider $\hat{f}_{\sigma}(x)=\breve{f}_{\sigma}(x)-\int_{0}^{1} \breve{f}_{\sigma}(x) d x . \quad \breve{f}_{\sigma}(x)=$ $(\sigma-|x-1 / 2|) / \sigma^{2} \chi_{[1 / 2-\sigma, 1 / 2+\sigma]}$, which is piecewise differentiable and often called a hat function or $C^{0}$ linear spline. In the second example, we consider some smooth right hand side functions given by Fourier modes $\tilde{f}_{k}(x)=\sin (k x \pi)$ with $k$ being an even positive integer. We consider the case with a finite and relatively small $k$ and the case where $k$ gets larger as the horizon parameter $\delta$ decreases, namely $k=1 / \delta$. The latter case allows us to make observations for oscillatory solutions. In the third example, we use a right hand side given by a box-potential, that is, $f_{\sigma}(x)=\dot{f}_{\sigma}(x)-\int_{0}^{1} \hat{f}_{\sigma}(x) d x$ where $\dot{f}_{\sigma}(x)=$ $1 /(2 \sigma) \chi[1 / 2-\sigma, 1 / 2+\sigma]$. Note that if we let $\sigma \rightarrow 0$, then the sequences $\left\{\hat{f}_{\sigma}\right\}$ and $\left\{f_{\sigma}\right\}$ all converge to the Dirac delta measure $f_{\star}(x)=\delta(1 / 2-x)-1$, which is a limiting case that we pay special attention to. We note that the sequence $\left\{\tilde{f}_{\sigma}\right\}$ has the function with constant value zero as the limit in the standard $L^{2}$ weak topology.

For all examples considered here, the local solution $u_{0}$ can be analytically constructed by solving the corresponding local problems which serves as a convenient benchmark. Similar constructions can be found in [38] for various choices of micromudulus or kernel functions. To quantify the errors, we mainly consider the pointwise $L^{\infty}$ norm in this section but results based on the discrete $L^{2}$ norm are also discussed in a number of instances. We adopt (2.13) to get the nonlocal solutions. The asymptotic compatibility of (2.13) has been carefully studied in [42] as well as the convergence of several limiting processes. Thus, the corresponding experimental observations are given only if some potential discrepancies need to be clarified. Our focus, naturally, is to compare the behavior of local and nonlocal discrete gradient operators defined by (2.15) both with fixed $\delta>0$ (for the solution of nonlocal models) and with $\delta \rightarrow 0$ (for bench-marking with well-established local solutions). 
Taking $h=\delta / r$ as the mesh size for an integer $r \geq 1$, we consider the following limiting behaviors: 1) let $h \rightarrow 0$ with a fixed $\delta$ to see if the nonlocal schemes are compatible with the continuum limits; 2) for a sufficiently small $h$ (so that we effectively have the nonlocal solutions available on the continuum level), reduce $\delta$ to see the $\delta$-dependence of the convergence of nonlocal solutions to the local limit; 3 ) let both $h$ and $\delta$ go to zero simultaneously with a fixed $r$, so as to test the asymptotic compatibility.

\subsection{The case of hat function $\hat{f}_{\sigma}$ with a given positive $\sigma$.}

In this example, we choose $\sigma=0.25$. In terms of the limiting behavior of numerical nonlocal solutions as $h \rightarrow 0$, we get results that are the same as in [42], namely numerical solutions $u_{\delta, h}$ of the nonlocal problem converge to $u_{\delta}$ at each grid point with an error on the order of $\mathcal{O}\left(h^{2}\right)$. Moreover, if $u_{0, h}$ denotes the numerical solution of the limiting local model on the same mesh, then

$$
\left\|u_{\delta, h}-u_{0, h}\right\|_{\infty} \sim \mathcal{O}\left(\delta^{2}\right)
$$

meaning that the discrete nonlocal scheme is asymptotically compatible with the local limit. The error between the discrete solutions of the nonlocal and local problems is on the order of the modeling error. Numerical data are omitted. On the other hand, the data collected on the local derivatives tell us that

$$
\left\|\mathbb{G}_{0}\left(u_{\delta}-u_{0}\right)\right\|_{\infty} \sim \mathcal{O}\left(\delta^{2}\right),
$$

see the columns 2 and 3 of Table 1 . Note that at the jump discontinuity of $G_{0} u_{\delta}$, the averaged value across the discontinuity is used. The derivative data have not been presented before in [42] since the earlier work was primarily concerned with the numerical solution only, not its derivatives. The second order convergence in the derivatives is due to the regularity pickup resulted from the fact that the local and nonlocal operators under consideration commute with each other for the periodic boundary condition case.

By applying the nonlocal gradient operator $\mathbb{G}_{\delta, h}$, instead of the local one, we find that

$$
\left\|\mathbb{G}_{\delta, h} u_{\delta, h}-\mathcal{R}_{h} \mathbb{G}_{\delta} u_{\delta}\right\|_{\infty} \sim \mathcal{O}\left(h^{2}\right)
$$

where $\mathcal{R}_{h}$ is the restriction to the quadrature points $\left\{x_{i}=i h\right\}_{i=0}^{N}$, as shown in the second and third columns of Table 2. Moreover,

$$
\left\|\mathbb{G}_{\delta} u_{\delta}-\mathbb{G}_{0} u_{0}\right\|_{\infty} \sim \mathcal{O}\left(\delta^{2}\right),
$$

as presented in columns 4 and 5 of Table 1 . The asymptotically compatible property of discrete nonlocal gradient schemes can also be observed, for example, by letting $h \rightarrow 0$ with a fixed $\delta / h$. Columns 4 and 5 of Table 2 show that the error order as $h \rightarrow 0$ with the fixed $\delta / h=4$ is $\mathcal{O}\left(h^{2}\right)$ as expected. We provide some error estimate of $\mathbb{G}_{\delta, h} u_{\delta, h}-\mathbb{G}_{0, h} u_{0, h}$ in Theorem 6 of the next section for $\eta \geq 1$ to further substantiate the experimental findings. 


\begin{tabular}{|c|c|c|c|c|}
\hline$\delta$ & $\left\|\mathbb{G}_{0} u_{\delta}-\mathbb{G}_{0} u_{0}\right\|_{\infty}$ & Order & $\left\|\mathbb{G}_{\delta} u_{\delta}-\mathbb{G}_{0} u_{0}\right\|_{\infty}$ & Order \\
\hline $2^{-2}$ & $1.60 \times 10^{-2}$ & -- & $5.96 \times 10^{-2}$ & -- \\
\hline $2^{-3}$ & $3.76 \times 10^{-3}$ & 2.08 & $1.42 \times 10^{-2}$ & 2.07 \\
\hline $2^{-4}$ & $9.25 \times 10^{-4}$ & 2.02 & $3.54 \times 10^{-3}$ & 2.00 \\
\hline $2^{-5}$ & $2.30 \times 10^{-4}$ & 2.00 & $8.85 \times 10^{-4}$ & 2.00 \\
\hline $2^{-6}$ & $5.75 \times 10^{-5}$ & 2.00 & $2.21 \times 10^{-4}$ & 2.00 \\
\hline
\end{tabular}

Table 1: $L^{\infty}$ errors of local and nonlocal gradient operators as $\delta$ decreases with $f=\hat{f}_{\sigma}$.

\begin{tabular}{|c|c|c|c|c|}
\hline & \multicolumn{2}{|c|}{ fixed $\delta=1 / 4$} & \multicolumn{2}{c|}{ fixed $\delta / h=4$} \\
\hline$h$ & $\left\|\mathbb{G}_{\delta, h} u_{\delta, h}-\mathcal{R}_{h} \mathbb{G}_{\delta, \frac{h}{2}} u_{\delta, \frac{h}{2}}\right\|_{\infty}$ & Order & $\left\|\mathbb{G}_{\delta, h} u_{\delta, h}-\mathcal{R}_{h} \mathbb{G}_{0} u_{0}\right\|_{\infty}$ & Order \\
\hline $2^{-4}$ & $6.85 \times 10^{-4}$ & -- & $3.41 \times 10^{-3}$ & -- \\
\hline $2^{-5}$ & $1.70 \times 10^{-4}$ & 2.01 & $8.52 \times 10^{-4}$ & 2.00 \\
\hline $2^{-6}$ & $4.23 \times 10^{-5}$ & 2.00 & $2.13 \times 10^{-4}$ & 2.00 \\
\hline $2^{-7}$ & $1.06 \times 10^{-5}$ & 2.00 & $5.32 \times 10^{-5}$ & 2.00 \\
\hline $2^{-8}$ & $2.64 \times 10^{-6}$ & 2.00 & $1.33 \times 10^{-5}$ & 2.00 \\
\hline
\end{tabular}

Table 2: $L^{\infty}$ errors of discrete nonlocal gradient as $h \rightarrow 0$ with a fixed $\delta$ or a fixed $\delta / h$ for $f=\hat{f}_{\sigma}$.

In comparison, we can also apply the special nonlocal gradient operator $\mathbb{G}_{\delta}^{p}$ as well as its discrete forms $\mathbb{G}_{\delta, h}^{p}$ or $\mathbb{G}_{\delta, h}^{p \pm}$. Since the exactness of nonlocal gradients at the discrete level is guaranteed in Theorem 2 for any $\delta$, we will focus on the limiting processes of vanishing $h$ with a fixed $\delta$ or fixed $\delta / h$. In the second and third columns of Table $3, \mathbb{G}_{\delta, h}^{p+}$ is used and $\mathbb{G}_{\delta, h}^{p+} u_{\delta, h}$ converges to $\mathbb{G}_{\delta}^{p} u_{\delta}=\mathbb{G}_{0} u_{0}$ as shown in Theorem 1, on the order of $\mathcal{O}(h)$ at each grid point. Moreover, when we use the discrete form $\mathbb{G}_{\delta, h}^{p}$, in principle we expect second order convergence, the same as the convergence order of local central difference schemes to local models as mentioned in Theorem 3. However, superconvergence can be observed for the difference approximation of local solutions, yielding exact grid point values of derivatives, see the last column of Table 3. One can compare the two kinds of nonlocal gradient operators by Table 2 and Table 3 . Both schemes converge to their continuum nonlocal gradients, $\mathbb{G}_{\delta} u_{\delta}$ and $\mathbb{G}_{\delta}^{p} u_{\delta}$, where the former has $\mathcal{O}\left(\delta^{2}\right)$ error from its local limit $\mathbb{G}_{0} u_{0}$ but the latter is exactly the local derivatives.

\subsection{The case of Fourier modes $\tilde{f}_{k}$.}

While the first example gives the $O\left(\delta^{2}\right)$ error order of nonlocal gradient recovery, there is no quantitative estimates on how the modeling data affect the error. For $\tilde{f}_{k}(x)=\sin (k \pi x)$ with $k=2$ and let $\delta \rightarrow 0$, we expect the second order in accuracy, same as the example 1 (see Columns 2 and 3 of Table 4). However, if we let $k=1 / \delta$ and let $\delta \rightarrow 0$, we see that the error order of the 


\begin{tabular}{|c|c|c|c|}
\hline$h$ & $\left\|\mathbb{G}_{\delta, h}^{p+} u_{\delta, h}-\mathcal{R}_{h} \mathbb{G}_{\delta}^{p+} u_{\delta}\right\|_{\infty}$ & Order & $\left\|\mathbb{G}_{\delta, h}^{p} u_{\delta, h}-\mathcal{R}_{h} \mathbb{G}_{\delta}^{p} u_{\delta}\right\|_{\infty}$ \\
\hline $2^{-4}$ & $9.38 \times 10^{-2}$ & -- & $5.55 \times 10^{-17}$ \\
\hline $2^{-5}$ & $4.69 \times 10^{-2}$ & 1.00 & $2.22 \times 10^{-16}$ \\
\hline $2^{-6}$ & $2.34 \times 10^{-2}$ & 1.00 & $4.44 \times 10^{-16}$ \\
\hline $2^{-7}$ & $1.17 \times 10^{-2}$ & 1.00 & $8.88 \times 10^{-16}$ \\
\hline $2^{-8}$ & $5.86 \times 10^{-3}$ & 1.00 & $2.66 \times 10^{-15}$ \\
\hline
\end{tabular}

Table 3: $L^{\infty}$ errors and error order of the special nonlocal gradient operator as $h \rightarrow 0$ with a fixed $\delta=1 / 4$ for $f=\hat{f}_{\sigma}$.

nonlocal gradient is one order less than that in first example (see columns 4 and 5 of Table 4 ), due to the $\delta$-dependent highly oscillatory right hand side. Such a reduction of error order matches with the analysis in Theorem 6 corresponding to cases $\eta=1$ and 0 .

\begin{tabular}{|c|c|c|c|c|}
\hline & \multicolumn{2}{|c|}{$k=2$} & \multicolumn{2}{c|}{$k=1 / \delta$} \\
\hline$\delta$ & $\left\|\mathbb{G}_{\delta} u_{\delta}-\mathbb{G}_{0} u_{0}\right\|_{\infty}$ & Order & $\left\|\mathbb{G}_{\delta} u_{\delta}-\mathbb{G}_{0} u_{0}\right\|_{\infty}$ & Order \\
\hline $2^{-2}$ & $1.97 \times 10^{-2}$ & -- & $3.98 \times 10^{-2}$ & -- \\
\hline $2^{-3}$ & $4.92 \times 10^{-3}$ & 2.01 & $1.99 \times 10^{-2}$ & 1.00 \\
\hline $2^{-4}$ & $1.23 \times 10^{-3}$ & 2.00 & $9.95 \times 10^{-3}$ & 1.00 \\
\hline $2^{-5}$ & $3.07 \times 10^{-4}$ & 2.00 & $4.97 \times 10^{-3}$ & 1.00 \\
\hline $2^{-6}$ & $7.67 \times 10^{-5}$ & 2.00 & $2.49 \times 10^{-3}$ & 1.00 \\
\hline
\end{tabular}

Table 4: $L^{\infty}$ errors and error orders between nonlocal gradient of nonlocal solutions and derivatives of the local limits as $\delta$ decreases for source terms $\left\{\tilde{f}_{k}\right\}$ with $k=2$ or $k=1 / \delta$.

\subsection{The case of box-potential $f_{\sigma}$ with a positive $\sigma$.}

The case of discontinuous $f_{\sigma}$ with $\sigma=0.25$ serves to compare the performances of local and nonlocal gradient schemes. Since $f_{\sigma}$ is not in $H^{1}$, the nonlocal solution $u_{\delta}$ also fails to be in $H^{1}$. For a fixed $\delta$ and vanishing $h$, the conventional local gradient operator fails to provide useful information in stress analysis, which is shown in Figure 1. This is true regardless which local discrete derivative operator, among forward, backward and central differences, is used. Table 5 shows the divergence of local gradient of nonlocal solutions for a fixed $\delta$ and vanishing $h$ in the discrete $L^{2}$ norm. Hence, for a fixed $\delta$, local gradient schemes are neither effective nor compatible when the solutions lose regularity.

Figure 1: The conventional local gradient of nonlocal solutions for discontinuous data with a fixed $\delta=0.1$ as $h \rightarrow 0$. Top: $h=0.01$; middle: $h=0.002$; bottom: $h=0.001$. X-axis: the periodic cell $\Omega=[0,1]$; Y-axis: values of local gradients. 


\begin{tabular}{|c|c|c|}
\hline & central difference & forward/backward difference \\
\hline$h$ & $\left\|\mathbb{G}_{0, h} u_{\delta, h}-\mathcal{R}_{h} \mathbb{G}_{0, \frac{h}{2}} u_{\delta, \frac{h}{2}}\right\|_{L^{2}}$ & $\left\|\mathbb{G}_{0, h}^{ \pm} u_{\delta, h}-\mathcal{R}_{h} \mathbb{G}_{0, \frac{h}{2}}^{ \pm} u_{\delta, \frac{h}{2}}\right\|_{L^{2}}$ \\
\hline $2^{-5}$ & 0.118 & 0.167 \\
\hline $2^{-6}$ & 0.167 & 0.236 \\
\hline $2^{-7}$ & 0.236 & 0.333 \\
\hline $2^{-8}$ & 0.333 & 0.471 \\
\hline $2^{-9}$ & 0.471 & 0.667 \\
\hline
\end{tabular}

Table 5: Divergence of discrete local gradient schemes of nonlocal solutions with a fixed $\delta=0.25$ as $h \rightarrow 0$.

As for the nonlocal gradients, one can compare Figure 1 with Figure 2 to see the differences between the local and nonlocal schemes with a fixed $\delta$ and vanishing $h$. Since the nonlocal solutions are only piecewise differentiable in this case, local derivatives blow up at certain grid points as mesh gets refined. Thus, local gradient based stress analysis would not produce useful information. However, if we apply the nonlocal gradient operator on the same nonlocal solution, the corresponding nonlocal gradient not only stays bounded but also, as shown in Figure 3, offers compatibility to the local derivative of the local limit as $\delta \rightarrow 0$. In this particular case, the maximum stress points coincide with those leading to maximum stress points associated to the local model.

Figure 2: The nonlocal gradient recovery of discontinuous data with a fixed $\delta=0.1$ as $h \rightarrow 0$. Top: $h=0.01$; middle: $h=0.002$; bottom: $h=0.001$. X-axis: the periodic cell $\Omega=[0,1]$; $\mathrm{Y}$-axis: values of nonlocal gradients.

In Figure 3, we choose a fixed but a sufficiently small $h$ (so that the modeling error is dominating) and let $\delta$ decrease, we can find that the nonlocal gradient of $u_{\delta}$ converges to the local derivative of $u_{0}$. In fact, the results in Table 6 lead to

$$
\left\|\mathbb{G}_{\delta} u_{\delta}-\mathbb{G}_{0} u_{0}\right\|_{\infty} \sim \mathcal{O}(\delta)
$$

and

$$
\left\|\mathbb{G}_{\delta} u_{\delta}-\mathbb{G}_{0} u_{0}\right\|_{L^{2}} \sim \mathcal{O}\left(\delta^{3 / 2}\right) .
$$

The $L^{2}$ error order matches with what is stated in Theorem 6 in the next section corresponding to $\eta=1 / 2$.

Figure 3: The nonlocal gradient recovery for discontinuous data with a sufficiently small $h=0.001$ as $\delta$ decreases. Top: $\delta=0.1$; middle: $\delta=0.05$; bottom: $\delta=0.025$. X-axis: the periodic cell $\Omega=[0,1]$; Y-axis: values of nonlocal gradients and limiting local gradients. 


\begin{tabular}{|c|c|c|c|c|}
\hline & \multicolumn{2}{|c|}{$L^{\infty}$ norm } & \multicolumn{2}{c|}{$L^{2}$ norm } \\
\hline$\delta$ & $\left\|\mathbb{G}_{\delta} u_{\delta}-\mathbb{G}_{0} u_{0}\right\|_{\infty}$ & Order & $\left\|\mathbb{G}_{\delta} u_{\delta}-\mathbb{G}_{0} u_{0}\right\|_{L^{2}}$ & Order \\
\hline $2^{-2}$ & $7.40 \times 10^{-2}$ & -- & $2.57 \times 10^{-2}$ & -- \\
\hline $2^{-3}$ & $3.72 \times 10^{-2}$ & 0.99 & $9.14 \times 10^{-3}$ & 1.49 \\
\hline $2^{-4}$ & $1.86 \times 10^{-2}$ & 1.00 & $3.23 \times 10^{-3}$ & 1.50 \\
\hline $2^{-5}$ & $9.31 \times 10^{-3}$ & 1.00 & $1.14 \times 10^{-3}$ & 1.50 \\
\hline $2^{-6}$ & $4.66 \times 10^{-3}$ & 1.00 & $4.10 \times 10^{-4}$ & 1.49 \\
\hline
\end{tabular}

Table 6: $L^{\infty}$ and $L^{2}$ errors and error orders between nonlocal gradient of nonlocal solutions and derivatives of the local limits as $\delta$ decreases with the right hand side being $f_{\sigma}$.

In addition, we can also compare $\mathbb{G}_{\delta, h}$ with $\mathbb{G}_{\delta, h}^{p}$ at the discrete level. The results are presented in Table 7. Again, both schemes converge to the corresponding nonlocal gradients $\mathbb{G}_{\delta} u_{\delta}$ and $\mathbb{G}_{\delta}^{p} u_{\delta}$ on the order of $\mathcal{O}(h)$ at each grid point, but the former has $L^{\infty}$ error from $\mathbb{G}_{0} u_{0}$ on the order of $\mathcal{O}(\delta)$ as shown in Table 6 while the latter is exactly the local derivatives as shown in Theorem 1.

\begin{tabular}{|c|c|c|c|c|}
\hline$h$ & $\left\|\mathbb{G}_{\delta, h} u_{\delta, h}-\mathcal{R}_{h} \mathbb{G}_{\delta, \frac{h}{2}} u_{\delta, \frac{h}{2}}\right\|_{\infty}$ & Order & $\left\|\mathbb{G}_{\delta, h}^{p} u_{\delta, h}-\mathcal{R}_{h} \mathbb{G}_{\delta}^{p} u_{\delta}\right\|_{\infty}$ & Order \\
\hline $2^{-4}$ & $7.44 \times 10^{-3}$ & -- & $3.13 \times 10^{-2}$ & -- \\
\hline $2^{-5}$ & $3.74 \times 10^{-3}$ & 1.00 & $1.56 \times 10^{-2}$ & 1.00 \\
\hline $2^{-6}$ & $1.90 \times 10^{-3}$ & 0.98 & $7.81 \times 10^{-3}$ & 1.00 \\
\hline $2^{-7}$ & $9.63 \times 10^{-4}$ & 0.98 & $3.91 \times 10^{-3}$ & 1.00 \\
\hline $2^{-8}$ & $4.85 \times 10^{-4}$ & 0.99 & $1.95 \times 10^{-3}$ & 1.00 \\
\hline
\end{tabular}

Table 7: $L^{\infty}$ errors and error order of two nonlocal gradient operators as $h \rightarrow 0$ with a fixed $\delta=1 / 4$ for $f=f_{\sigma}$.

\subsection{The case of Dirac-Delta function $f_{\star}$.}

For the previous example, as $\sigma \rightarrow 0$, the right hand side becomes a Dirac delta function, which cannot be interpolated onto grid points directly. We may first mollify the right hand side via a convolution with a kernel also parametrized by $\delta$. Certainly the mollification could in general have a different scaling factor $\sigma$, and this will be discussed more in the next section. Different kernels also lead to different mollification $\tilde{f}_{\star}$. For example, $\tilde{f}_{\star}$ can be $f_{\delta}$ or $\hat{f}_{\delta}$ that are identical to $f_{\sigma}$ and $\hat{f}_{\sigma}$ with $\sigma$ replaced by $\delta$. In this experiment we use these two choices to do mollifications, namely, the right hand side data are the same as in two of the previous examples but are parametrized by the horizon. As $\delta$ is reduced, when the mesh is fine enough (so that the discretization error is much less significant than the modeling error), we can make comparisons of the nonlocal solutions with local limits as well as nonlocal gradient with derivatives of its local limit. 
First, we use the mollification $\hat{f}_{\delta}$ and let $\tilde{u}_{\delta}$ denote the solution of mollified nonlocal problems. Table 8 represents the error order of $\left|\tilde{u}_{\delta}-u_{0}\right|$ in $L^{2}$ norm. A theoretical analysis can be found in Theorem 5 corresponding to $\beta=0$ and $\eta=-1 / 2$ where a Gaussian mollifier is used as an illustration. We note that $\hat{f}_{\delta}$ is regular enough so that it behaves similarly like a Gaussian in the limit.

\begin{tabular}{|c|c|c|}
\hline$\delta$ & $\left\|\tilde{u}_{\delta}-u_{0}\right\|_{L^{2}}$ & Order \\
\hline $2^{-2}$ & $5.73 \times 10^{-3}$ & -- \\
\hline $2^{-3}$ & $2.09 \times 10^{-3}$ & 1.45 \\
\hline $2^{-4}$ & $7.50 \times 10^{-4}$ & 1.48 \\
\hline $2^{-5}$ & $2.67 \times 10^{-4}$ & 1.49 \\
\hline $2^{-6}$ & $9.49 \times 10^{-5}$ & 1.50 \\
\hline
\end{tabular}

Table 8: $L^{2}$ errors and error orders between nonlocal solutions of the mollified equation and the local limits as $\delta$ decreases with a Diract-delta function right hand side subject to an $H^{1}$ mollification.

Now we turn to the two gradient recovery techniques with different mollifications. Since $\hat{f}_{\delta} \in H^{1}$, the nonlocal solution of the mollified equation, denoted by $\tilde{u}_{\delta}$, is also in $H^{1}$. Both local and nonlocal gradient schemes converge to the local derivative of $u_{0}$ in the sense of $L^{2}$ norm. Figure 4 shows such limiting behavior of the nonlocal gradient operator as $\delta \rightarrow 0$ and Table 9 establishes the error orders of the two gradient schemes. Again we refer to Theorem 6 for related theoretical analysis with $\eta=-1 / 2$. The maximum nonlocal stress happens at the location of the point load, again the same as the case for local limit.

Figure 4: The nonlocal gradient recovery for a mollified Dirac-Delta function data as $\delta$ decreases. X-axis: the periodic cell $\Omega=[0,1]$; Y-axis: values of nonlocal gradients with different $\delta$.

\begin{tabular}{|c|c|c|c|c|}
\hline & \multicolumn{2}{|c|}{ local gradient } & \multicolumn{2}{c|}{ nonlocal gradient } \\
\hline$\delta$ & $\left\|\mathbb{G}_{0} \tilde{u}_{\delta}-\mathbb{G}_{0} u_{0}\right\|_{L^{2}}$ & Order & $\left\|\mathbb{G}_{\delta} \tilde{u}_{\delta}-\mathbb{G}_{0} u_{0}\right\|_{L^{2}}$ & Order \\
\hline $2^{-2}$ & $1.13 \times 10^{-1}$ & -- & $1.80 \times 10^{-1}$ & -- \\
\hline $2^{-3}$ & $8.02 \times 10^{-2}$ & 0.50 & $1.27 \times 10^{-1}$ & 0.50 \\
\hline $2^{-4}$ & $5.65 \times 10^{-2}$ & 0.50 & $8.96 \times 10^{-2}$ & 0.50 \\
\hline $2^{-5}$ & $4.00 \times 10^{-2}$ & 0.51 & $6.31 \times 10^{-2}$ & 0.51 \\
\hline $2^{-6}$ & $2.79 \times 10^{-2}$ & 0.51 & $4.43 \times 10^{-2}$ & 0.51 \\
\hline
\end{tabular}

Table 9: $L^{2}$ errors and error orders between local and nonlocal gradients and derivatives of local solutions as $\delta$ decreases with a Dirac-delta function data subject to an $H^{1}$ mollification.

In comparison, if we pick $f_{\delta}$ as the mollified right hand side, the local gradient operator does not provide useful information as mentioned in the earlier 


\begin{tabular}{|c|c|c|}
\hline$\delta$ & $\left\|\mathbb{G}_{\delta} \tilde{u}_{\delta}-\mathbb{G}_{0} u_{0}\right\|_{L^{2}}$ & Order \\
\hline $2^{-2}$ & $1.83 \times 10^{-1}$ & -- \\
\hline $2^{-3}$ & $1.38 \times 10^{-1}$ & 0.41 \\
\hline $2^{-4}$ & $1.00 \times 10^{-1}$ & 0.45 \\
\hline $2^{-5}$ & $7.19 \times 10^{-2}$ & 0.48 \\
\hline $2^{-6}$ & $5.10 \times 10^{-2}$ & 0.49 \\
\hline
\end{tabular}

Table 10: $L^{2}$ errors and error orders between nonlocal gradients and derivatives of local solutions as $\delta$ decreases with the Dirac-delta function subject to an $L^{2}$ mollification.

experiment due to the lack of regularity of $u_{\delta}$. However, the nonlocal gradient operator still works and offers the same error order as when we use $\hat{f}_{\delta}$, see data in Table 10.

We omit the case for $\mathbb{G}_{\delta}^{p} \tilde{u}_{\delta}$ as we get the same superconvergence predicted by the earlier theorems.

\subsection{Discussions on experiments}

The numerical experiments reported above use quadrature based approximations and cover various types of data and solutions. We make a brief summary based on the experimental observations. First, by comparing local gradient operators and generic nonlocal gradient operators $\mathbb{G}_{\delta}$ as well as their discretization, we find that

1. For smooth enough $\left(C^{1}\right.$ or $H^{1}$ or better) data where local gradient operators can be directly used to calculate the derivatives of $u_{\delta}$, nonlocal gradient operators also perform well, especially when both $h \rightarrow 0$ and $\delta \rightarrow 0$.

2. For $H^{\alpha}$ data with $\alpha \in[0,1)$, local gradient fails to provide credible information in nonlocal stress analysis, whereas nonlocal gradient schemes still perform well and possess the asymptotically compatible property.

3. For $H^{\alpha}$ data with $\alpha \in(-1,0)$, one may mollify the right hand first in order to do quadrature collocation approximations. Then nonlocal gradient operators work well in recovering the derivatives of local limits but whether local gradient operators work or not depends on the regularity of mollified right hand data.

These findings provide motivations to the theoretical analysis given in the next section.

While the nonlocal gradient operators performs better than local one for conducting stress analysis, different nonlocal gradient operators, such as $\mathbb{G}_{\delta, h}$ and $\mathbb{G}_{\delta, h}^{p}$, can also perform differently. On one hand, $\mathbb{G}_{\delta, h}$ gives us the freedom of 
choosing the kernel $\beta_{\delta}$ and also works well in other kinds of volume-constrained problems that are nonlocal analog of boundary value problems, see $[11,12]$ for the connections. On the other hand, while $\mathbb{G}_{\delta, h}^{p}$ is a specialized operator under our considerations of $1 \mathrm{~d}$ periodic model and quadrature based discretization, it has some unique features. Apart from the AC property, $\mathbb{G}_{\delta, h}^{p}$ can recover exactly the difference of the local solution as $h$ goes to 0 with any $\delta$, as shown both theoretically in Theorem 1 and 2 and numerically in the experiments above. The study of $\mathbb{G}_{\delta}^{p}$ in multidimensional cases and discrete forms for other numerical methods, for instance Galerkin finite element methods, will be given in future works.

\section{Some theoretical analysis}

We provide some theoretical insights to the observed convergence results based on numerical experiments, We adopt the Fourier analysis due to the periodic boundary condition, similar to the analysis of spectral methods given in $[46,15]$.

\subsection{Preliminaries}

Suppose the number of grid points is $2 N$ on the interval $[0,1]$ with spacing $h=\frac{1}{2 N}$. Now for a periodic array $\left\{u_{j}\right\}$ defined on the grid, we use the discrete Fourier transform: for $j=1,2, \cdots, 2 N$, and $n=-N+1, \cdots, 1, \cdots, N$,

$$
u_{j}=\frac{1}{2 \pi} \sum_{n=-N+1}^{N} e^{i 2 \pi n x_{j}} \hat{u}_{n} \quad \text { and } \quad \hat{u}_{n}=h \sum_{j=1}^{2 N} e^{-i 2 \pi n x_{j}} u_{j} .
$$

Let us take $\delta=r h$ for an integer $r \geq 1$ for simplicity right now. Let $U_{N}=\left(u_{1}, u_{2}, \ldots, u_{N}\right)^{T}$ and $F_{N}=\left(f_{1}, f_{2}, \ldots, f_{N}\right)^{T}$. For any $\eta \in \mathbb{R}$, we take $\|\cdot\|_{\eta}$ as the discrete $H^{\eta}$ norm and $|\cdot|_{\eta}$ as the discrete semi- $H^{\eta}$ norm defined by

$\left|U_{N}\right|_{\eta}^{2}=h \sum_{j=1}^{2 N}|n|^{2 \eta}\left|u_{j}\right|^{2}=\frac{1}{2 \pi} \sum_{\substack{n=-N+1 \\ n \neq 0}}^{N}|n|^{2 \eta}\left|\hat{u}_{n}\right|^{2} \quad$ and $\quad\left\|U_{N}\right\|_{\eta}^{2}=\left|U_{N}\right|_{\eta}^{2}+\left\|U_{N}\right\|_{0}^{2}$

with $\|\cdot\|_{0}$ being the standard $L^{2}$ norm.

First, $\left\{\left(e^{i 2 \pi n x_{1}}, e^{i 2 \pi n x_{2}}, \cdots, e^{i 2 \pi n x_{N}}\right)^{T}\right\}$ forms an eigenbasis of the discrete operators $\mathbb{A}_{\delta}^{h}, \mathbb{A}^{h}, \mathbb{G}_{\delta, h}$ and $\mathbb{G}_{0, h}$ introduced before, with eigenvalues given respectively by

$$
\lambda_{\delta}^{h}(n)=2 \sum_{j=1}^{r} b_{j}(1-\cos (2 \pi n j h)), \quad \lambda^{h}(n)=\frac{2}{h^{2}}(1-\cos (2 \pi n h)) .
$$


for integer $n$ between 1 and $N$ where $b_{j}$ 's are given as in (2.14) and

$$
\mu_{\delta}^{h}(n)=i h \sum_{j=1}^{r} j b_{j} \sin (2 \pi n j h), \quad \mu^{h}(n)=\frac{i}{h} \sin (2 \pi n h) .
$$

As in the experiments corresponding to a non-smooth right hand side $f \in H^{\eta}$ for $-1<\eta<0$, we introduce a mollifier on $f$ to enhance its regularity, and thus $u_{\delta}$ simultaneously. We only consider the Gaussian mollifier here as an illustration and leave more general cases to future discussions. Let $\mathcal{M}_{\delta}$ be the Gaussian mollifier defined by

$$
\mathcal{M}_{\delta} f(x)=\int_{-\infty}^{\infty} G_{\delta}(x-\tau) f(\tau) d \tau, \quad \text { where } \quad G_{\delta}(s)=\frac{1}{2 \delta \sqrt{\pi}} e^{-\left(\frac{s}{2 \delta}\right)^{2}} .
$$

One can define $\tilde{F}_{N}^{\delta}$ as: for $j=1,2, \cdots, N$,

$$
\left(\tilde{F}_{N}^{\delta}\right)_{j}=\mathcal{M}_{\delta} f\left(x_{j}\right)=\frac{1}{2 \pi} \sum_{\substack{n=-N+1 \\ n \neq 0}}^{N} e^{-4 \pi^{2} n^{2} \delta^{2}} e^{i 2 \pi n x_{j}} \hat{f}_{n} .
$$

\subsection{Convergence analysis of nonlocal numerical solutions with vanishing $\delta$}

We first verify the asymptotic compatibility, similar to the results of [15].

Theorem 4. Let $\mathbb{A}_{\delta}^{h} U_{N}^{\delta}=F_{N}$ and $\mathbb{A}^{h} U_{N}^{0}=F_{N}$, then

$$
\left\|U_{N}^{\delta}-U_{N}^{0}\right\|_{0} \leq C \delta^{2}\left\|F_{N}\right\|_{0},
$$

for a constant $C$ independent of $\delta, N$ and $F_{N}$.

Proof. It is easy to see that $U_{N}^{\delta}-U_{N}^{0}=\left(\left(\mathbb{A}_{\delta}^{h}\right)^{-1}-\left(\mathbb{A}^{h}\right)^{-1}\right) F_{N}$. So,

$$
\left\|U_{N}^{\delta}-U_{N}^{0}\right\|_{0}^{2}=\frac{1}{2 \pi} \sum_{\substack{n=-N+1 \\ n \neq 0}}^{N}\left|\frac{1}{\lambda_{\delta}^{h}(n)}-\frac{1}{\lambda^{h}(n)}\right|^{2}\left|\hat{f}_{n}\right|^{2} .
$$

Thus, (4.22) follows from

$$
\frac{1}{\delta^{2}}\left|\frac{1}{\lambda_{\delta}^{h}(n)}-\frac{1}{\lambda^{h}(n)}\right| \leq C \quad \text { for } 1 \leq|n| \leq N,
$$

which is shown in the Lemma 7 in the appendix.

Next, if $f \in H^{\eta}$ for some $\eta$ such that $-1 \leq \eta<0$, we consider the mollification operator $\mathcal{M}_{\delta}$ defined in (4.20). Denote by $\tilde{U}_{N}^{\delta}$ and $\tilde{U}_{N}^{0}$ the solutions to following mollified equations respectively:

$$
\mathbb{A}_{\delta}^{h} \tilde{U}_{N}^{\delta}=\tilde{F}_{N}^{\delta}, \quad \mathbb{A}^{h} \tilde{U}_{N}^{0}=\tilde{F}_{N}^{\delta},
$$

where $\tilde{F}_{N}^{\delta}$ is defined in (4.21). Then we have the following theorem with vanishing $\delta$. 
Theorem 5. Assume that $F_{N} \in H^{\eta}$ with $-1<\eta<0$. Then for any $0 \leq \beta \leq$ $2+\eta$

$$
\left\|\tilde{U}_{N}^{\delta}-U_{N}^{0}\right\|_{\beta} \leq C \delta^{2+\eta-\beta}\left|F_{N}\right|_{\eta},
$$

where $C$ is a constant independent of $N$ and $\delta$.

Proof. First of all, $\left\|\tilde{U}_{N}^{\delta}-U_{N}^{0}\right\|_{\beta} \leq\left\|\tilde{U}_{N}^{\delta}-\tilde{U}_{N}^{0}\right\|_{\beta}+\left\|\tilde{U}_{N}^{0}-U_{N}^{0}\right\|_{\beta}$ and

$$
\begin{aligned}
\left\|\tilde{U}_{N}^{0}-U_{N}^{0}\right\|_{\beta}^{2} & =\left\|\left(\mathbb{A}^{h}\right)^{-1}\left(\tilde{F}_{N}^{\delta}-F_{N}\right)\right\|_{\beta}^{2} \\
& =\frac{1}{2 \pi} \sum_{\substack{n=-N+1 \\
n \neq 0}}^{N}\left(\left|\lambda^{h}(n)\right|^{-2}|n|^{-2 \eta+2 \beta}\left|e^{-4 \pi^{2} n^{2} \delta^{2}}-1\right|^{2}\right)|n|^{2 \eta}\left|\hat{f}_{n}\right|^{2} .
\end{aligned}
$$

Next we aim to show $\left|\lambda^{h}(n)\right|^{-1} \leq c|n|^{-2}$ by following

$$
\begin{aligned}
\left|\lambda^{h}(n)\right| & =\frac{h^{2}}{2(1-\cos (2 \pi n h))}=\frac{1}{4 \pi^{2}} \frac{(n \pi h)^{2}}{\sin ^{2}(n \pi h)}|n|^{-2} \\
& \leq \frac{1}{4 \pi^{2}}|n|^{-2} \frac{(\pi / 2)^{2}}{\sin ^{2}(\pi / 2)}=\frac{1}{16}|n|^{-2},
\end{aligned}
$$

where in the last step we have used the fact that function $\frac{\theta}{\sin (\theta)}$ is monotonically increasing in $(0, \pi / 2]$ and $|n \pi h| \in(\pi / N, \pi / 2]$ for $n \neq 0$. On the other hand, from Taylor expansion, it can be easily checked that for any $|n| \geq 1$,

$$
|n|^{-2-\eta+\beta}\left|e^{-4 \pi^{2} n^{2} \delta^{2}}-1\right| \leq C \delta^{2+\eta-\beta} .
$$

Combing (4.25) and (4.26) gives

$$
\left\|\tilde{U}_{N}^{0}-U_{N}^{0}\right\|_{\beta} \leq C \delta^{2+\eta-\beta}\left|F_{N}\right|_{\eta},
$$

Meanwhile, for the first term, we have

$$
\left\|\tilde{U}_{N}^{\delta}-\tilde{U}_{N}^{0}\right\|_{\beta}^{2} \leq \frac{C}{2 \pi} \sum_{\substack{n=-N+1 \\ n \neq 0}}^{N}\left(|n|^{2 \beta} e^{-8 \pi^{2} n^{2} \delta^{2}}\left|\frac{1}{\lambda_{\delta}^{h}(n)}-\frac{1}{\lambda^{h}(n)}\right|^{2}\right)\left|\hat{f}_{n}\right|^{2} .
$$

Again we can check that $|n|^{-\eta+\beta} e^{-4 \pi^{2} n^{2} \delta^{2}} \leq C \delta^{\eta-\beta}$ for any $|n| \geq 1$. Together with (4.23), we arrive at

$$
\left\|\tilde{U}_{N}^{\delta}-\tilde{U}_{N}^{0}\right\|_{\beta} \leq C \delta^{2+\eta-\beta}\left|F_{N}\right|_{\eta},
$$

which completes the proof.

Remark 1. We can take different horizon parameters in nonlocal diffusion operators $\left(\delta_{1}\right)$ and mollification operators $\left(\delta_{2}\right)$. Then instead of $(4.24)$, we can get

$$
\left\|\tilde{U}_{N}^{\delta_{1}}-U_{N}^{0}\right\|_{\beta} \leq C\left(\delta_{1}^{2} \delta_{2}^{\eta-\beta}+\delta_{2}^{2+\eta-\beta}\right)\left|F_{N}\right|_{\eta} .
$$

More details are given in a subsequent work [13]. 


\subsection{Convergence analysis of nonlocal gradient recovery}

We now show the asymptotic compatibility of the nonlocal gradient schemes for the kernel defined in (3.18).

Theorem 6. Assume that $U_{N}^{\delta}$ and $U_{N}^{0}$ are solutions to $\mathbb{A}_{\delta}^{h} U_{N}^{\delta}=F_{N}$ and $\mathbb{A}^{h} U_{N}^{0}=F_{N}$ respectively. Then

$$
\left\|\mathbb{G}_{\delta, h} U_{N}^{\delta}-\mathbb{G}_{0, h} U_{N}^{0}\right\|_{0} \leq C \delta^{\min \{2,1+\eta\}}\left|F_{N}\right|_{\min \{\eta, 1\}},
$$

where $C$ is a constant independent of $N$ and $\delta$.

The proof of the theorem is given in the appendix.

Remark 2. One can choose two different horizon parameters in nonlocal diffusion operator $\left(\delta_{1}\right)$ and nonlocal gradient operator $\left(\delta_{2}\right)$. Then instead of (4.29), we have

$$
\left\|\mathbb{G}_{\delta_{2}, h} U_{N}^{\delta_{1}}-\mathbb{G}_{0, h} U_{N}^{0}\right\|_{0} \leq C\left(\frac{\delta_{1}^{2}}{\delta_{2}^{\max \{0,1-\eta\}}}+\delta_{2}^{\min \{2,1+\eta\}}\right)\left|F_{N}\right|_{\min \{\eta, 1\}} .
$$

We refer to [13] for detailed discussions.

\section{Discussions and conclusions}

Robust algorithms and analysis are essential for predictive multiscale simulations. For complex systems treating defects and cracks such as those modeled by peridynamics, algorithms that are sensitive to changes of parameters may produce results that are unphysical or difficult to verify/validate [42, 43]. In this work, we have provided both numerical evidence and some preliminary theoretical substantiation to the robustness of the nonlocal gradient recovery using asymptotically compatible discretizations. This, through appropriate constitutive relation, can lead to effective nonlocal stress analysis, Let us now offer some additional discussions on the use of nonlocal gradient operators in nonlocal modeling.

First, the notions of peridynamic nonlocal stress and nonlocal strains have been extensively discussed in [34]. Simple ways are suggested to match nonlocal concepts with their classical local analog. Advantages and limitations have been further investigated in subsequent works [21, 6, 44]. Most recently, in [20], a nonlocal strain measure was developed for noisy DIC measurement. It is formulated as a post-processing technique of measurement or simulation data so that there is no direct link made to the use of such strain measure within nonlocal modeling itself. The computation of such a measure was also made on pixel levels of DIC images that may or may not be tied to the numerical discretizations of the underlying continuum mechanical models. Indeed, it is 
easy for one to see the advantage of nonlocal gradients on the analysis of noisy images or less regular quantities. Similar observations can also be found in [9] for processing noisy images. We note, however, that the work presented here attempts to explore and connect the nonlocality present in both numerical discretizations and the underlying physical processes,

The notion of nonlocal gradient may also be related to the use of kernelbased integral approximations to differential operators in methods like SPH and RKPM $[22,28]$. Although many forms of the integral relaxations of differential equations have been around in the literature for a few decades, there are much fewer careful studies of the continuum forms of these nonlocal operators, along with discussions on the appropriate function spaces or domains of definition and properties of these nonlocal integral operators before they get discretized. The situation has started to change recently. $[12,27]$ attempted to provide a rigorous and systematic framework. Meanwhile, some nonlocal derivatives and gradients discussed in the literature, defined either directly on discrete levels or when discretized, only involve function values of particles within a fixed multiple of typical particle spacings (much like $\delta \leq r h$ for a fixed $r$ in our context). Technically such discrete operators should only be viewed as approximating local differential operators. This is the case even if the defined gradients depend on more than just the nearest neighbors. The discrete nonlocal operators studied here intrinsically involve two separate length scales, one that characterizes the range of nonlocality on the continuum level while the other serves as a depiction of particle spacing which is dependent on the level of numerical resolution. The concept of asymptotical compatibility is again important in that it allows robust estimations of nonlocal gradients and their local counterpart in respective regimes. It also allows us to delineate the roles of physical scale and level of numerical resolutions.

Our work here demonstrates that while nonlocal gradients may need to be used with caution in correspondence theory [34, 6, 44], they can be utilized as gradient recovery techniques or nonlocal stress analysis. For clarity, we have limited our theoretical illustration to a special nonlocal model problem in one dimension using quadratures based collocation methods, which have close resemblance with the popular particle based meshfree methods used for peridynamics. To make the main point clear, we have considered only the simple one dimensional case with periodic boundary conditions. The techniques can obviously be extended to more complex systems in higher dimensions and under suitable constraints due to the geometric boundary. In such cases, we may adopt the following more general forms of nonlocal gradient operators, as defined in [27] with a given third-order odd tensor kernel $\mathcal{K}_{\delta}$ and a specified scalar kernel $\beta_{\delta}$,

$$
\mathcal{G}_{\delta} \mathbf{u}(\mathbf{x}):=\lim _{\epsilon \rightarrow 0} \int_{\Omega \backslash B_{\epsilon}(\mathbf{x})} \beta_{\delta}(|\mathbf{y}-\mathbf{x}|) \mathcal{K}_{\delta}(\mathbf{y}-\mathbf{x}) \mathcal{D}(\mathbf{u})(\mathbf{y}, \mathbf{x}) d \mathbf{y}
$$

for any vector field $\mathbf{u}$ defined on a bounded domain $\Omega$ where $B_{\epsilon}(\mathbf{x})$ is a ball centered at a point $\mathbf{x}$ with radius $\epsilon$ and $\mathcal{D}(\mathbf{u})(\mathbf{y}, \mathbf{x}):=(\mathbf{u}(\mathbf{y})-\mathbf{u}(\mathbf{x})) /|\mathbf{y}-\mathbf{x}|$. This continuum form extends those defined in [12]. While it appears to be 
much more involved than the scalar version (and in the absence of physical boundary), we may draw analogy with the path towards the development of $\mathrm{AC}$ schemes. Indeed, the discussion on AC schemes was first started with simple one dimensional scalar equations [42] which subsequently have been generalized to multiple dimensions and for systems of nonlocal models [14, 43] as well as nonlinear models [15]. Along this line, more refined mathematical theory also gets established based on the theoretical analysis [13]. The special choices of the kernels also allow us to draw connections with PD stress tensor [34] that further enable us to introduce mixed method formulation as another approach for nonlocal gradient recovery. These topics and applications to simulations of cracks and defects using nonlocal models are subjects of ongoing studies.

Acknowledgement: The authors would like to thank S. Silling, M. Gunzburger, R. Lehoucq, J. Foster, M. Parks, J.S. Chen, W.K. Liu, D. Turner and in particular T. Mengesha, for discussions on related subjects.

\section{Appendix}

We first complete the proof of theorem 6 then present a lemma used for showing the theorem 4 .

Proof of Theorem 6. For $\eta \geq 1$, it is easy to verify (4.29). For $-1<\eta<1$, we prove the inequality by

$$
\left\|\mathbb{G}_{\delta, h} U_{N}^{\delta}-\mathbb{G}_{0, h} U_{N}^{0}\right\|_{0} \leq\left\|\mathbb{G}_{\delta, h} U_{N}^{\delta}-\mathbb{G}_{\delta, h} U_{N}^{0}\right\|_{0}+\left\|\mathbb{G}_{\delta, h} U_{N}^{0}-\mathbb{G}_{0, h} U_{N}^{0}\right\|_{0} .
$$

Furthermore, we aim to show

$$
\left\|\mathbb{G}_{\delta, h} U_{N}^{\delta}-\mathbb{G}_{\delta, h} U_{N}^{0}\right\|_{0} \leq C \delta^{1+\eta}\left|F_{N}\right|_{\eta},
$$

and

$$
\left\|\mathbb{G}_{\delta, h} U_{N}^{0}-\mathbb{G}_{0, h} U_{N}^{0}\right\|_{0} \leq C \delta^{1+\eta}\left|F_{N}\right|_{\eta} .
$$

It suffices to show that for all $n$ such that $1 \leq|n| \leq N$,

$$
R_{n}:=\delta^{-1-\eta}|n|^{-\eta}\left|\frac{\mu_{\delta}^{h}(n)}{\lambda_{\delta}^{h}(n)}-\frac{\mu_{\delta}^{h}(n)}{\lambda^{h}(n)}\right| \leq C
$$

and

$$
T_{n}:=\delta^{-1-\eta}|n|^{-\eta}\left|\frac{\mu_{\delta}^{h}(n)}{\lambda^{h}(n)}-\frac{\mu^{h}(n)}{\lambda^{h}(n)}\right| \leq C .
$$

In each part, we separate the cases $\delta|n| \leq 1 / 2$ and $\delta|n|>1 / 2$. For $R_{n}$, we rewrite it as

$$
R_{n}=\left(\delta^{1-\eta}|n|^{-\eta}\left|\mu_{\delta}^{h}(n)\right|\right)\left(\frac{1}{\delta^{2}}\left|\frac{1}{\lambda_{\delta}^{h}(n)}-\frac{1}{\lambda^{h}(n)}\right|\right) .
$$


Due to (4.23), it suffices to show

$$
\delta^{1-\eta}|n|^{-\eta}\left|\mu_{\delta}^{h}(n)\right| \leq C .
$$

When $\delta|n| \leq 1 / 2$, since $|\sin (2 \pi n j h)| \leq 2 \pi|n| j h$,

$$
\left|\mu_{\delta}^{h}(n)\right| \leq 2 \pi|n| \sum_{j=1}^{r} b_{j}(j h)^{2} .
$$

By the fact that $\sum_{j=1}^{r} b_{j}(j h)^{2}=1$,

$$
\delta^{1-\eta}|n|^{-\eta}\left|\mu_{\delta}^{h}(n)\right| \leq 2 \pi(\delta|n|)^{1-\eta} \leq 2^{\eta} \pi .
$$

When $\delta|n|>1 / 2$, one can calculate that

$$
b_{j}=\frac{3 h}{\delta^{3}}, \quad j=1,2, \cdots, r-1,
$$

and

$$
b_{r}=\frac{h}{\delta^{3}}\left(\frac{3}{2}-\frac{h}{2 \delta}\right) .
$$

Since $r \geq 1,1 \leq \frac{3}{2}-\frac{h}{2 \delta}<\frac{3}{2}$. Therefore,

$$
\left|\mu_{\delta}^{h}(n)\right| \leq \frac{C h^{2}}{\delta^{3}}\left|\sum_{j=1}^{r} j \sin (2 \pi n j h)\right| .
$$

Moreover, we claim that

$$
\left|\sum_{j=1}^{r} j \sin (2 \pi n j h)\right| \leq \frac{C \delta}{n h^{2}} .
$$

Indeed, let $a=2 \pi n h$, our goal is to show

$$
\left|\sum_{j=1}^{r} j \sin (j a)\right| \leq \frac{C r}{a} \quad \forall a \in[0, \pi], \forall r \geq 1 .
$$

By the formula $2 \sin (a j) \sin (a)=\cos (a(j-1))-\cos (a(j+1))$, we can rewrite 
$\sum_{j=1}^{r} j \sin (j a)$ as

$$
\begin{aligned}
\sum_{j=1}^{r} j \sin (j a) & =\frac{1}{2 \sin (a)} \sum_{j=1}^{r} j[\cos (a(j-1))-\cos (a(j+1))] \\
& =\frac{1}{2 \sin (a)}\left(\cos (0)+2 \sum_{j=1}^{r} \cos (a j)-(r+1) \cos (a r)-r \cos (a(r+1))\right) \\
& =\frac{1}{2 \sin (a)}\left(1+\frac{\sin ((r+1 / 2) a)-\sin (a / 2)}{\sin (a / 2)}-(r+1) \cos (a r)-r \cos (a(r+1))\right) \\
& =\frac{1}{2 \sin (a)}\left(\frac{\sin ((r+1 / 2) a)}{\sin (a / 2)}-(r+1) \cos (a r)-r(\cos (a r) \cos (a)-\sin (a r) \sin (a))\right) \\
& =\frac{1}{2 \sin (a)}\left(\frac{\cos (a(r+1))-\cos (a r)}{-2 \sin ^{2}(a / 2)}-(r \cos (a)+r+1) \cos (a r)+r \sin (a r) \sin (a)\right) \\
& =\frac{1}{2 \sin (a)}\left(-(r \cos (a)+r) \cos (a r)+\frac{\sin (a)}{1-\cos (a)} \sin (a r)+r \sin (a r) \sin (a)\right) .
\end{aligned}
$$

Hence

$$
\left|\sum_{j=1}^{r} j \sin (j a)\right| \leq \frac{r(1+\cos (a))}{2 \sin (a)}+\frac{|\sin (a r)|}{2(1-\cos (a))}+\frac{1}{2} r|\sin (a r)| .
$$

Now we split $a \in[0, \pi]$ into two cases. For $a \in[0,3]$, there exist $c_{1}, c_{2}>0$ such that

$$
\begin{aligned}
\sin (a) & \geq c_{1} a \\
1-\cos (a) & \geq c_{2} a^{2} .
\end{aligned}
$$

Then

$$
\left|\sum_{j=1}^{r} j \sin (j a)\right| \leq \frac{r}{c_{1} a}+\frac{a r}{2 c_{2} a^{2}}+\frac{1}{2} r \leq C \frac{r}{a} .
$$

For $a \in[3, \pi]$, there exists $\tilde{c}_{1}>0$ such that

$$
\begin{aligned}
\sin (a) & \geq \tilde{c}_{1}(\pi-a) \\
1+\cos (a) & \leq \frac{1}{2}(\pi-a)^{2}
\end{aligned}
$$

through Taylor expansions around $\pi$. So

$$
\left|\sum_{j=1}^{r} j \sin (j a)\right| \leq \frac{r(\pi-a)^{2}}{4 \tilde{c}_{1}(\pi-a)}+\frac{a r}{2}+\frac{1}{2} r \leq \tilde{C} r \leq C \frac{r}{a} .
$$

We then can get

$$
\delta^{1-\eta}|n|^{-\eta}\left|\mu_{\delta}^{h}(n)\right| \leq C(\delta|n|)^{-1-\eta} \leq 2^{1+\eta} C .
$$


For $T_{n}$, we first claim that $\left|\mu_{\delta}^{h}(n)\right| \leq\left|\mu^{h}(n)\right|$. Indeed, one can prove by induction that

$$
|\sin (2 \pi n j h)| \leq j|\sin (2 \pi n h)|
$$

for any positive integer $j$. Therefore,

$$
\left|\sum_{j=1}^{r} j h b_{j} \sin (2 \pi n j h)\right| \leq \frac{1}{h} \sum_{j=1}^{r}(j h)^{2} b_{j}|\sin (2 \pi n h)|=\frac{1}{h}|\sin (2 \pi n h)|,
$$

which implies our claim. Now, we rewrite $T_{n}$ as

$$
T_{n}=\delta^{-1-\eta}|n|^{-\eta}\left|\frac{\mu^{h}(n)}{\lambda^{h}(n)}\right|\left|\frac{\mu_{\delta}^{h}(n)}{\mu^{h}(n)}-1\right| .
$$

We have that

$$
\left|\frac{\mu^{h}(n)}{\lambda^{h}(n)}\right| \leq \frac{C}{|n|} \quad \forall h,
$$

where $C$ is a constant that is independent of $h$. This can be verified by the basic inequalities $|\sin (\theta)| \leq|\theta|$ and $2(1-\cos (\theta)) \geq \theta^{2}-\theta^{4} / 12$. Therefore, it suffices to show

$$
(\delta|n|)^{-1-\eta}\left|\frac{\mu_{\delta}^{h}(n)}{\mu^{h}(n)}-1\right| \leq C .
$$

When $\delta|n| \leq 1 / 2$, since $\sin (\theta)>\theta-\theta^{3} / 6$, we have

$$
1-\frac{\mu_{\delta}^{h}(n)}{\mu^{h}(n)} \leq 1-\frac{2 \pi n-(2 \pi n)^{3} \delta^{2} / 6}{2 \pi n}=(2 \pi n \delta)^{2} / 6,
$$

thus

$$
(\delta|n|)^{-1-\eta}\left|\frac{\mu_{\delta}^{h}(n)}{\mu^{h}(n)}-1\right| \leq C(\delta|n|)^{1-\eta} \leq C .
$$

We can also check the case $\delta|n|>1 / 2$ since

$$
\left|\frac{\mu_{\delta}^{h}(n)}{\mu^{h}(n)}-1\right| \leq\left|\frac{\mu_{\delta}^{h}(n)}{\mu^{h}(n)}\right|+1 \leq 2 .
$$

Next, we present a technical result used in the proof of theorem 4 .

Lemma 7. There exists a positive constant $C$ independent of $N, h$ and $\delta$, such that

$$
\frac{1}{\delta^{2}}\left|\frac{1}{\lambda_{\delta}^{h}(n)}-\frac{1}{\lambda^{h}(n)}\right| \leq C \quad \text { for } 1 \leq|n| \leq N .
$$


Proof. First, $\mathbb{A}_{\delta}^{h}$ is exactly $\mathbb{A}^{h}$ when $\delta \leq h([42])$, so we only show the result for $\delta>h$.

For $\delta|n| \leq 1 / 2$, by $\theta^{2}-\theta^{4} / 12 \leq 2(1-\cos (\theta)) \leq \theta^{2}$ and the fact that

$$
\sum_{j=1}^{r} b_{j}(j h)^{2}=1 \quad \text { and } \quad \sum_{j=1}^{r} b_{j}(j h)^{4} \leq \delta^{2} \sum_{j=1}^{r} b_{j}(j h)^{2}=\delta^{2},
$$

we can show that

$$
\begin{array}{r}
0<(2 \pi n \delta)^{2}-\frac{(2 \pi n \delta)^{4}}{12} \leq \delta^{2} \lambda_{\delta}^{h}(n) \leq(2 \pi n \delta)^{2}, \\
0<(2 \pi n \delta)^{2}-\frac{(2 \pi n \delta)^{4}}{12} \leq(2 \pi n \delta)^{2}-\frac{(2 \pi n)^{4} \delta^{2} h^{2}}{12} \leq \delta^{2} \lambda^{h}(n) \leq(2 \pi n \delta)^{2} .
\end{array}
$$

Therefore,

$$
\frac{1}{\delta^{2}}\left|\frac{1}{\lambda_{\delta}^{h}(n)}-\frac{1}{\lambda^{h}(n)}\right| \leq \frac{1}{(2 \pi n \delta)^{2}-\frac{(2 \pi n \delta)^{4}}{12}}-\frac{1}{(2 \pi n \delta)^{2}} \leq \frac{1}{12-\pi^{2}} .
$$

Now when $\delta|n|>1 / 2$, let us show that both $\frac{1}{\delta^{2} \lambda_{\delta}^{h}(n)}$ and $\frac{1}{\delta^{2} \lambda^{h}(n)}$ are uniformly bounded above. For the former, using $|n| h \leq N h=1 / 2$, the bound follows since we have

$$
\delta^{2} \lambda^{h}(n) \geq(2 \pi n \delta)^{2}\left(1-\frac{(2 \pi n h)^{2}}{12}\right) \geq \pi^{2}\left(1-\frac{\pi^{2}}{12}\right) .
$$

Considering $\delta^{2} \lambda_{\delta}^{h}(n)$, we split the sum in the expression for $\lambda_{\delta}^{h}(n)$ into two parts:

$$
\begin{aligned}
\delta^{2} \sum_{j=1}^{4 n j h \leq 1} b_{j}(1-\cos (2 \pi n j h)) & \geq\left((2 \pi n \delta)^{2}-\frac{(2 \pi n)^{4} \delta^{2}}{12(4 n)^{2}}\right) \sum_{j=1}^{4 n j h \leq 1} b_{j}(j h)^{2} \\
& \geq \pi^{2}\left(1-\frac{\pi^{2}}{48}\right) \sum_{j=1}^{4 n j h \leq 1} b_{j}(j h)^{2} .
\end{aligned}
$$

We can see that there exists a constant $c_{0}>0$ such that

$$
\delta^{2} \sum_{4 n j h>1}^{r} b_{j}(1-\cos (2 \pi n j h)) \geq c_{0} \delta^{2} \sum_{4 n j h>1}^{r} b_{j} \geq c_{0} \sum_{4 n j h>1}^{r} b_{j}(j h)^{2} .
$$

Therefore, the lemma is valid for all $n$.

[1] B. Alali, and M. Gunzburger, Peridynamics and material interfaces, Journal of Elasticity, 120.2 (2015), 225-248.

[2] F. Andreu, J. M. Mazon, J. D. Rossi, and J. Toledo, Nonlocal Diffusion Problems, Math. Surveys Monographs 165, AMS, Providence, RI, (2010). 
[3] D. Applebaum, Levy Processes and Stochastic Calculus, Cambridge Stud. Adv. Math., 93 ( 2004), Cambridge University Press, Cambridge, UK.

[4] E. Askari, F. Bobaru, R. B. Lehoucq, M. L. Parks, S. A. Silling, and O. Weckner, Peridynamics for multiscale materials modeling, J. Phys. Conf. Ser., 125 (2008), 12-78.

[5] P. W. Bates and A. Chmaj, An integrodifferential model for phase transitions: Stationary solutions in higher space dimensions, J. Statist. Phys., 95 (1999), 1119-1139.

[6] M. Bessa, J. Foster, T. Belytschko, and W. K. Liu, A meshfree unification: reproducing kernel peridynamics, Computational Mechanics, 53 (2014), 1251-1264.

[7] F. Bobaru and M. Duangpanya, The peridynamic formulation for transient heat conduction, Internat. J. Heat Mass Transfer, 53 (2010), 4047-4059.

[8] F. Bobaru, M. Yang, L. F. Alves, S. A. Silling, E. Askari, and J. Xu, Convergence, adaptive refinement, and scaling in $1 \mathrm{~d}$ peridynamics, Internat. $J$. Numer. Methods Engrg., 77 (2009), 852-877.

[9] A. Buades, B. Coll, and J. M. Morel, Image denoising methods. A new nonlocal principle, SIAM Rev., 52 (2010), 113-147.

[10] X. Chen and M. Gunzburger, Continuous and discontinuous finite element methods for a peridynamics model of mechanics, Comput. Methods Appl. Mech. Engrg., 200 (2011), 1237-1250.

[11] Q. Du, M. Gunzburger, R. B. Lehoucq, and K. Zhou, Analysis and approximation of nonlocal diffusion problems with volume constraints, SIAM Rev., 56 ( 2012), 676-696.

[12] Q. Du, M. Gunzburger, R. B. Lehoucq, and K. Zhou, A nonlocal vector calculus, nonlocal volume-constrained problems, and nonlocal balance laws, Math. Models Methods Appl. Sci., 23 (2013), 493-540.

[13] Q. Du, Y. Tao, X. Tian and J. Yang, Numerical analysis of nonlocal gradient recovery and its asymptotically compatible discretization, in preparation, (2016).

[14] Q. Du and X. Tian, Asymptotically compatible schemes for peridynamics based on numerical quadratures, ASME 2014 International Mechanical Engineering Congress and Exposition (IMECE2014), Vol.1 (2014), Paper No. IMECE2014-39620, pp. V001T01A058.

[15] Q. Du and J. Yang, Asymptotically compatible Fourier spectral approximations of nonlocal Allen-Cahn equations, to appear in SIAM J. Numerical Analysis, 2015. 
[16] Q. Du, J. Yang and Z. Zhou, Analysis of a nonlocal-in-time parabolic equation, submitted, 2016.

[17] Q. Du and K. Zhou, Mathematical analysis for the peridynamic nonlocal continuum theory, Math. Model. Numer. Anal., 45 (2011), 217-234.

[18] G. Gilboa and S. Osher,, Nonlocal operators with applications to image processing, Multiscale Model. Simul., 7 (2008), 1005-1028.

[19] B. Kilic and E. Madenci, Coupling of peridynamic theory and the finite element method, J. Mech. Materials Structures, 5 (2010), 707-733.

[20] R. Lehoucq, P. Reu, and D. Turner. A Nonlocal Strain Measure for Digital Image Correlation, Strain, 51 (2015), 265-275.

[21] R. Lehoucq, and S.A. Silling. Force flux and the peridynamic stress tensor, Journal of the Mechanics and Physics of Solids, 56.4 (2008), 1566-1577.

[22] W.K. Liu, Y. Chen, S. Jun, J. Chen, T. Belytschko, C. Pan, R. Uras and C. Chang, Overview and applications of the reproducing kernel particle methods, Archives of Computational Methods in Engineering, 3 (1996), 380 .

[23] Y. Lou, X. Zhang, S. Osher, and A. Bertozzi, Image recovery via nonlocal operators, J. Sci. Comput., 42 (2010), 185-197.

[24] R. Macek and S. A. Silling, Peridynamics via finite element analysis, Finite Elements Analy. Design, 43 (2007), 1169-1178.

[25] D. S. Malkus and T. J. Hughes, Mixed finite element methodsreduced and selective integration techniques: a unification of concepts, Computer Methods in Applied Mechanics and Engineering, 15 (1978), 63-81.

[26] T. Mengesha and Q. Du, Nonlocal Constrained Value Problems for a Linear Peridynamic Navier Equation, Journal of Elasticity, 116, (2014), 27-51.

[27] T. Mengesha and Q. Du, Characterization of function spaces of vector fields via nonlocal derivatives and an application in peridynamics, Nonlinear Analysis A: Theory, Methods and Applications, to appear, 2016.

[28] Monaghan, J.J., Smoothed particle hydrodynamics, Rep. Prog. Phys. 68 (2005), 1703-1759.

[29] G. Palatucci, O. Savin and E. Valdinoci, Local and global minimizers for a variational energy involving a fractional norm, Annali di matematica pura ed applicata, 192 (2012), 673-718.

[30] E. Oterkus and E. Madenci, Peridynamic analysis of fiber-reinforced composite materials, J. Mech. Materials Structures, 7 (2013), 45-84. 
[31] P.A. Raviart and J.M. Thomas. A mixed finite element method for 2-nd order elliptic problems. In Mathematical aspects of finite element methods, pp. 292-315. Springer Berlin Heidelberg, 1977.

[32] P. Seleson, M. Parks, M. Gunzburger, and R. Lehoucq, Peridynamics as an upscaling of molecular dynamics, Multiscale Model. Simul., 8 (2009), 204-227.

[33] J. Shen and T. Tang, Spectral and high-order methods with applications. Science Press, 2006.

[34] S.A. Silling, Reformulation of elasticity theory for discontinuities and longrange forces, J. Mech. Phys. Solids, 48 (2000), 175-209.

[35] S. A. Silling and E. Askari, A meshfree method based on the peridynamic model of solid mechanics, Comput. Structures, 83 (2005), 1526-1535.

[36] S. A. Silling and R. B. Lehoucq, Peridynamic theory of solid mechanics, Adv. Appl. Mech., 44 ( 2010), 73-168.

[37] S. A. Silling, O. Weckner, E. Askari, and F. Bobaru, Crack nucleation in a peridynamic solid, Internat. J. Fracture, 162 (2010), 219-227.

[38] S. A. Silling, M. Zimmermann, and R. Abeyaratne, Deformation of a peridynamic bar, Journal of Elasticity, 73 (2010), 173-190.

[39] E. Tadmor, Filters, mollifiers and the computation of the Gibbs phenomenon, Acta Numerica, 16 (2007), 305-378.

[40] E. Tadmor and C. Tan. Critical thresholds in flocking hydrodynamics with non-local alignment. Philosophical Transactions of the Royal Society of London A: Mathematical, Physical and Engineering Sciences, 372:20130401, 2014.

[41] H. Tian and L. Ju and Q. Du, Nonlocal convection-diffusion problems and finite element approximation, Comp. Meth. Appl. Mech. Engr, 289 (2015), 60-78.

[42] X. Tian and Q. Du, Analysis and comparison of different approximations to nonlocal diffusion and linear peridynamic equations, SIAM J. Numer. Anal., 51 (2013), 3458-3482.

[43] X. Tian and Q. Du, Asymptotically Compatible Schemes and Applications to Robust Discretization of Nonlocal Models, SIAM J. Numer. Anal., 52 (2014), 1641-1665.

[44] M.R. Tupek, R. Radovitzky. An extended constitutive correspondence formulation of peridynamics based on nonlinear bond-strain measures, Journal of the Mechanics and Physics of Solids, 65 (2014), 82-92. 
[45] O. Zienkiewicz and J. Zhu, The superconvergent patch recovery and a posteriori error estimates. Part 2: Error estimates and adaptivity. International Journal for Numerical Methods in Engineering, 33 (1992), 1365-1382.

[46] K. Zhou and Q. Du, Mathematical and numerical analysis of linear peridynamic models with nonlocal boundary conditions, SIAM J. Numer. Anal., 48 (2010), 1759-1780. 

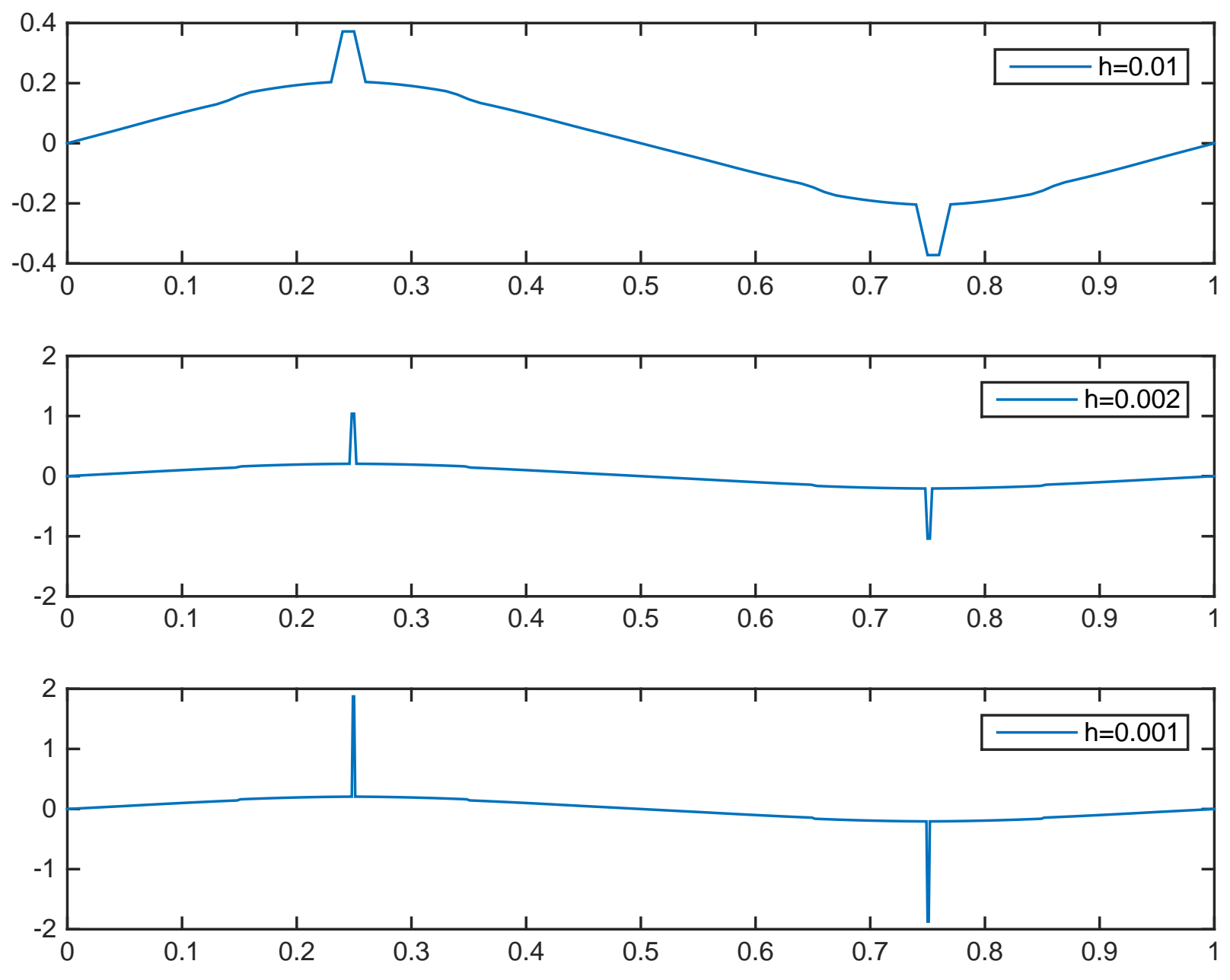

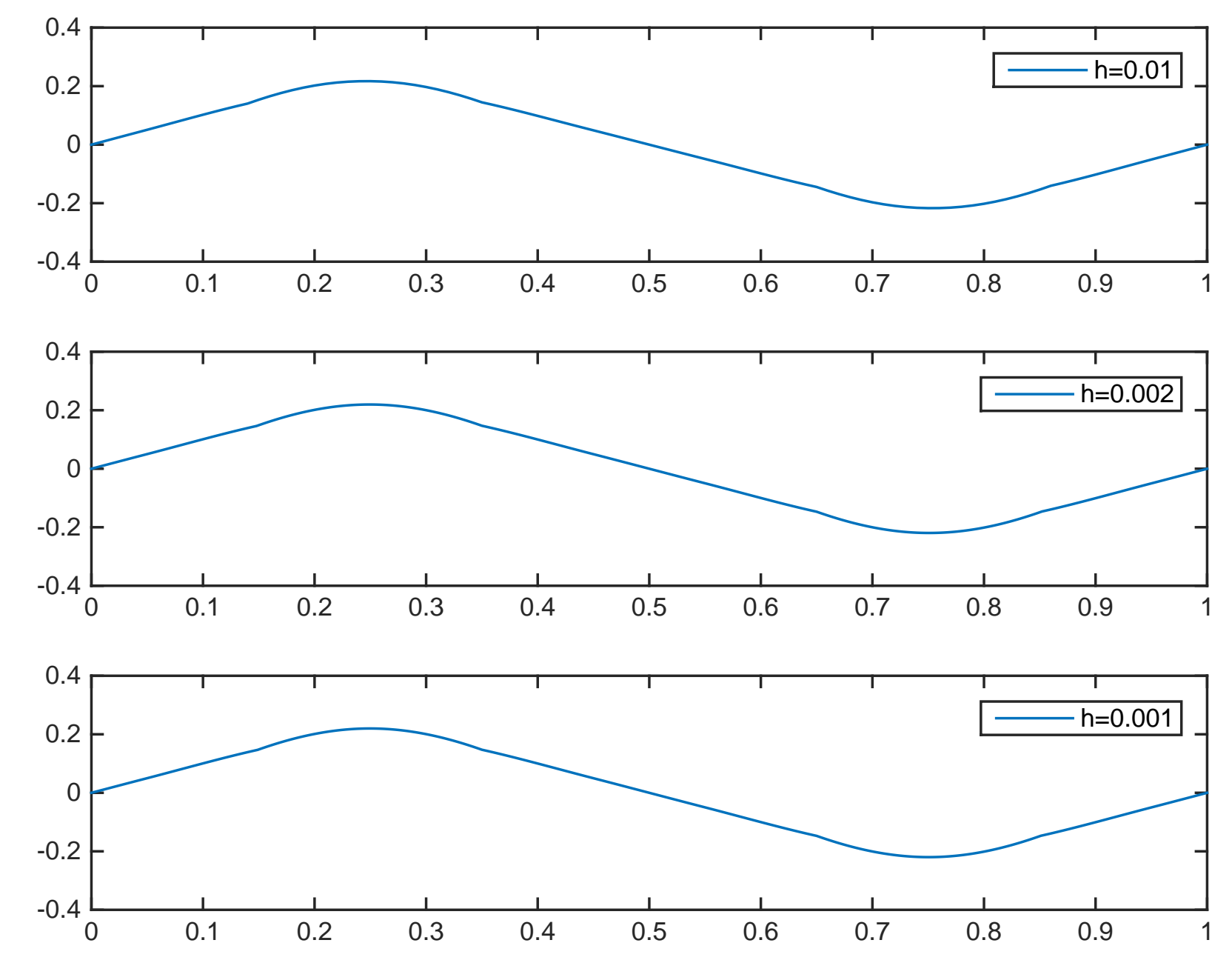

Figure 2 

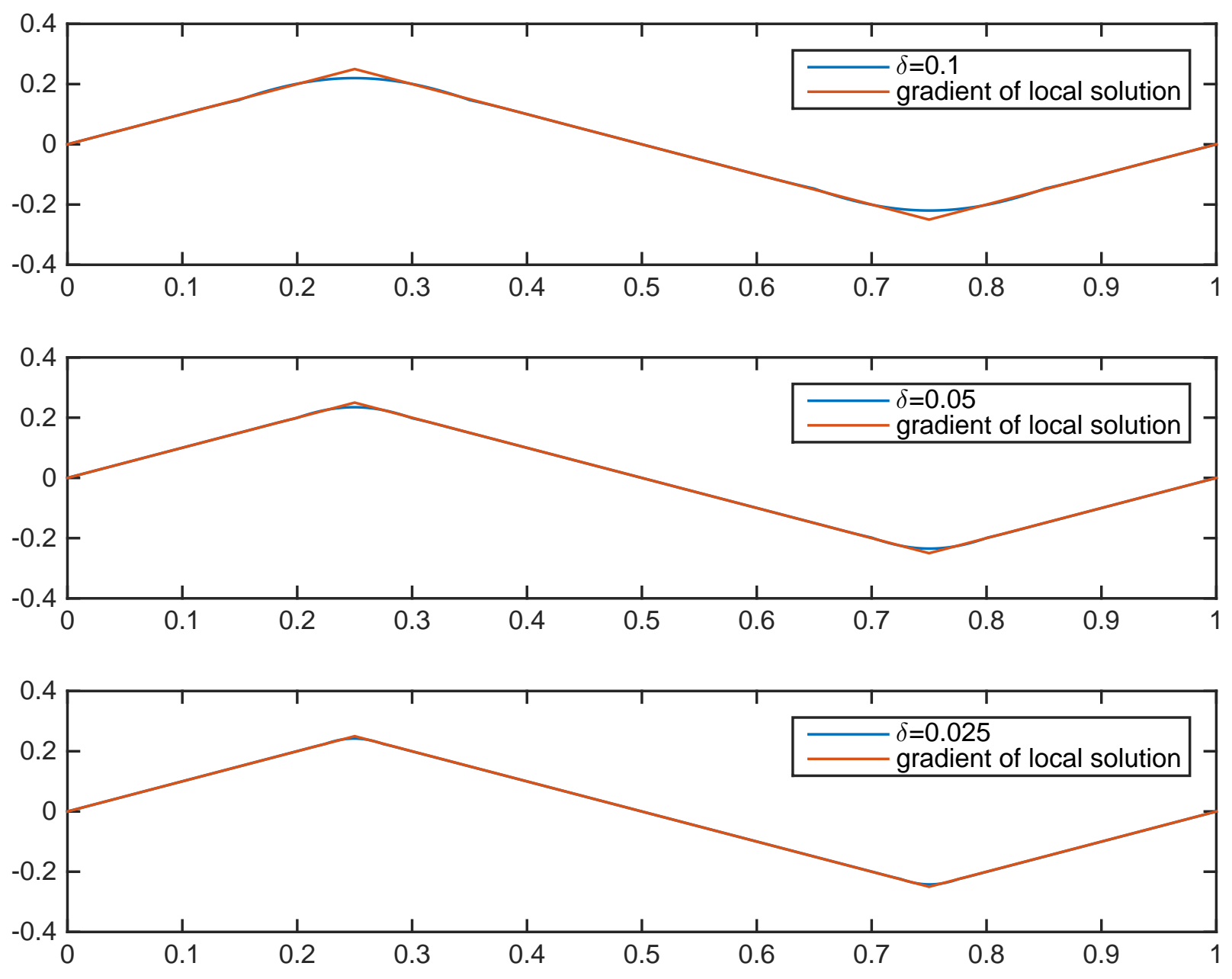
